\title{
Oxygenase Coordination Is Required for Morphological Transition and the Host-Fungus Interaction of Aspergillus flavus
}

\author{
Sigal Horowitz Brown, ${ }^{1}$ James B. Scott, ${ }^{1}$ Jeyanthi Bhaheetharan, ${ }^{2}$ William C. Sharpee, ${ }^{1}$ Lane Milde, ${ }^{1}$ \\ Richard A. Wilson, ${ }^{3}$ and Nancy P. Keller ${ }^{1,2,4}$ \\ ${ }^{1}$ Department of Plant Pathology and ${ }^{2}$ Department of Medical Microbiology and Immunology, University of Wisconsin-Madison, \\ 1550 Linden Dr., Madison 53706-1598, U.S.A.; ${ }^{3}$ Department of Plant Pathology, University of Nebraska, Lincoln, 68588, \\ U.S.A., and ${ }^{4}$ Department of Bacteriology, University of Wisconsin-Madison, Madison, U.S.A.
}

Submitted 10 November 2008. Accepted 28 March 2009.

Oxylipins, a class of oxygenase-derived unsaturated fatty acids, are important signal molecules in many biological systems. Recent characterization of an Aspergillus flavus lipoxygenase gene, lox, revealed its importance in maintaining a density-dependent morphology switch from sclerotia to conidia as population density increased. Here, we present evidence for the involvement of four more oxylipingenerating dioxygenases $(\mathrm{PpoA}, \mathrm{PpoB}, \mathrm{PpoC}$, and $\mathrm{PpoD})$ in A. flavus density-dependent phenomena and the effects of loss of these genes on aflatoxin production and seed colonization. Although several single mutants showed alterations in the sclerotia-to-conidia switch, the major effect was observed in a strain downregulated for all five oxygenases (invert repeat transgene [IRT] strain IRT4 $=p p o A, p p o B$, ppoC, ppoD, and lox). In strain IRT4, sclerotia production was increased up to 500 -fold whereas conidiation was decreased down to $\mathbf{1 0 0}$-fold and the strain was unable to switch into conidial production. Aflatoxin (AF) production for all mutant strains and the wild type was greatest at low population densities and absent in high populations except for strain IRT4, which consistently produced high levels of the mycotoxin. Growth on host seed by both IRT4 and IRT2 (downregulated in $p p o A, p p o B$, and $p p o D$ ) was marked by decreased conidial but increased AF production. We propose that $A$. flavus oxygenases and the oxylipins they produce act in a highly interdependent network with some redundancy of biological function. These studies provide substantial evidence for oxylipin-based mechanisms in governing fungus-seed interactions and in regulating a coordinated quorum-sensing mechanism in A. flavus.

Aspergillus flavus is an opportunistic pathogen of crops. It is important because it produces aflatoxin (AF) as a secondary metabolite in the seed of a number of crops both before and

S. H. Brown and J. B. Scott contributed equally to this study.

Corresponding author: Nancy Keller; Telephone: +1 (608) 262-9795; Fax: +1 (608) 262-8418; E-mail: npkeller@wisc.edu

Nucleotide sequences data reported here are available in the GenBank database under the accession numbers FJ263387 for ppoA; FJ263388 for ppoB; FJ263389 for ppoC; FJ263390 for $p p o D$.

*The $\boldsymbol{e}$-Xtra logo stands for "electronic extra" and indicates that three supplemental figures are published online. In addition, Figure 6 is in color online. after harvest. AF is a potent carcinogen that is highly regulated in most countries (Bhatnagar et al. 2006; Klich 2007; Robens and Cardwell 2003). A. flavus generates asexual spores (conidia) which serve as the primary disseminating and infectious unit. A. flavus also forms resting structures called sclerotia, hardened, pigmented mycelial tissues capable of long periods of dormancy during unfavorable growth conditions (Diener et al. 1987). Sclerotia-infected plant tissue such as corn kernels, cobs, and leaf tissue can remain in the soil and support the fungus until the following season when newly exposed mycelium or sclerotia can give rise to conidial structures, thus producing the primary inoculum for the next infection cycle (Diener et al. 1987; Hedayati et al. 2007; Scheidegger and Payne 2003).

Understanding how Aspergilli perceive environmental cues and adjust their morphological strategies is crucial for understanding how to address the agricultural and health damage they cause. Several known stimuli affect Aspergillus spp. development and reproduction, including temperature, $\mathrm{pH}$, carbon and nitrogen sources (Bennett et al. 1979; Cotty 1988; Gqaleni et al. 1997; Keller et al. 1997), air availability (Guzman-de-Peña and Ruiz-Herrera 1997), and the circadian oscillator (Greene et al. 2003). In addition, exogenous (seed-derived) and endogenous (Aspergillus-derived) signaling compounds called oxylipins also affect development in the Aspergilli (Calvo et al. 1999; Tsitsigiannis and Keller 2007; Tsitsigiannis et al. 2004).

Oxylipins are a broad class of oxygenated polyunsaturated fatty acids that function as molecular signals in plants, animals, insects, and fungi (Funk 2001). In fungi, oxylipins have emerged as key signals conserved among ecologically diverse groups. Oxylipins affect sporulation in Neurospora, Alternaria, and Aspergillus spp. (Champe et al. 1987; Hyeon 1976; Nukima et al. 1981) and toxin biosynthesis in Aspergillus spp. (Calvo et al. 2002; Tsitsigiannis and Keller 2006) and Fusarium spp. (McDonald et al. 2004); and act as pathogenicity-related signals for quorum sensing and phase transition in yeast and dimorphic fungi (Shea et al. 2006; Tsitsigiannis and Keller 2007). Corroborating these findings, recently it has been shown that $A$. flavus possesses a quorum-sensing-like mechanism, where a sclerotia-to-conidia transition is governed by cell density and lipoxygenase activity (Horowitz et al. 2008).

In plants, the enzymes responsible for forming oxylipins from polyunsaturated fatty acids are called lipoxygenases (LOX), which incorporate molecular oxygen into fatty acids at either the 9- or 13-carbon position (9-LOX and 13-LOX, respectively) (Liavonchanka and Feussner 2006; Nemchenko et al. 2006). In fungi, a family of lipid dioxgenases known as Ppo 
oxygenases are conserved in many filamentous fungi and produce oxylipins that are structurally and physiologically similar to those produced by plant lipoxygenases (Champe and ElZayat 1989; Champe et al. 1987; Hyeon 1976; Nukima et al. 1981; Tsitsigiannis and Keller 2007). Several lines of evidence suggest that plant and fungal oxylipins can functionally substitute for each other and are involved in fungus-host communications. Exogenously applied plant 9-LOX and 13-LOX linoleic acid-derived oxylipins have sporogenic capabilities comparable with the fungal-derived Ppo oxylipins (Calvo et al. 1999). Furthermore, 9-LOX-derived oxylipins increase mycotoxin biosynthesis, including AF, whereas 13-LOX-derived oxylipins inhibit biosynthesis (Burow et al. 1997), phenotypes reminiscent of specific ppo mutations in A. nidulans (Tsitsigiannis et al. 2006). Reciprocal "cross talk" between fungus and seed was also demonstrated when plant $L O X$ expression was altered in response to fungal infection in both peanut and maize seed (Burow et al. 2000; Tsitsigiannis et al. 2005b, 2006) and, furthermore, when peanut $L O X$ expression was muted when seed were infected by Aspergillus oxylipin mutants (Brodhagen et al. 2008). The response of Aspergillus spp. to seed oxylipins led to the postulation that host oxylipins can mimic or interfere with endogenous fungal oxylipins on a cellular basis, thus affecting fungal development and the outcome of the host-fungus interaction (Burow et al. 1997; Calvo et al. 1999). This was strengthened by experimental data when it was shown that expression of a maize 9-LOX gene in an A. nidulans Ppo mutant could partially remediate the $\triangle p p o$ phenotype (Brodhagen et al. 2008) and that a maize 9-LOX mutant was more susceptible to Aspergillus spp. infections (Gao et al. 2009).

Three Ppo dioxygenases have been characterized in the model organism A. nidulans. Deletion of $p p o A$ or $p p o B$ increases the ratio of asexual to sexual spores (Tsitsigiannis et al. $2004,2005 b)$ while deletion of ppoC has the opposite phenotype-an increase in sexual spores and decrease in asexual spores (Tsitsigiannis et al. 2005b). In the human pathogen $A$. fumigatus, there was no measurable phenotype in the ppoA and $p p o B$ mutants but the $p p o C$ mutant showed several abnormalities, including decreased spore production, increased conidial size, and altered host macrophage interactions (Dagenais et al. 2008). Considering the importance of Ppos in A. nidulans and A. fumigatus biology and Lox in A. flavus cell density morphology transitions (Horowitz et al. 2008), we set out to determine whether the four A. flavus ppos ( $p p o A, p p o B, p p o C$, and $p p o D$ ) were important in fungal development and pathogenicity. An examination of mutants containing a single ppo deletion as well as strains downregulated in expression of multiple ppo genes combined with lox loss, enabled assessment of the relative contribution of each gene to spore, sclerotial, and AF production as well as host colonization. The most striking phenotype was the strain deficient in all five oxygenases which resulted in blockage of the sclerotia-to-conidia transition and highly upregulated AF production. We propose that A. flavus Ppos and the oxylipins they produce participate in a quorumdirected developmental shift and act in a redundant but highly interdependent network that affects differentiation processes and the outcome of the host-fungus interaction.

\section{RESULTS}

\section{A. flavus genome contains four ppo fatty acid peroxidases.}

Four Ppo homologs of $A$. nidulans PpoA were identified in A. flavus genomic scaffolds. PpoA and PpoC are on chromosome 2 and $P p o B$ and PpoD are found on chromosomes 8 and 4 , respectively. Each Ppo contains a heme peroxidase domain as determined by the National Center for Biotechnology Information conserved domain search. Biology Workbench software was used to align A. flavus Ppo proteins based on predicted amino acid sequence identity with Ppos from A. nidulans and A. fumigatus. Percent sequence identities are shown in supplementary data (Supplementary Fig. 1). The PpoA and PpoC groups were highly conserved and contained homologs from each of the three species; the PpoB and PpoD groups were more loosely related, although strong pairing was seen between $A$. flavus and A. fumigatus.

\section{The ppo genes are expressed}

\section{during vegetative growth and reproduction.}

Because both $A$. nidulans and A. fumigatus ppo genes have been associated with sporulation processes, we examined expression of each A. flavus gene during conidiophore formation (Fig. 1). Only ppoA is expressed prior to conidiophore initiation. Its expression fluxgate during conidiophore formation but remains present from the beginning of vesicle formation and through the point when mature conidiophores appear. ppoC expression is detectable when the vesicles begin to produce metulae and gradually increases as mature conidia are produced. $p p o D$ becomes detectable as the first conidiophores mature and, finally, $p p o B$ is expressed when the bulk of conidiophores are bearing mature conidia (Fig. 1A). Relative expression of each ppo gene during conidiophore formation is presented in Figure 1B.

\section{Identification and characterization of oxygenase deletion mutants and RNAi-deficient mutants.}

To assess the functions of ppo genes in A. flavus, ppoA, $p p o C$, and $p p o D$ were inactivated separately by homologous recombination with the plasmids pJBS3.1, pRAW2.2, and pJBS4.1 in the $p y r G^{-}$strain NRRL 3357.5 (despite several tries, we were unable to delete $p p o B$ ). Southern analysis of transformants showed the replacement of each gene with $A$. parasiticus pyrG (Fig. 2A through $\mathrm{C}$ ). Initially, three transformants of each genotype were examined and, when preliminary physiology experiments showed similar phenotypes, one transformant of each genotype was selected for further tests: TJBS3.1.19 for the $\triangle p p o A$ genotype, TRAW2.2.15 for $\triangle p p o C$, and TJBS4.1.85 for $\triangle p p o D$. These three strains were compared with TSHB2.39 (Alox mutant) (Horowitz et al. 2008) and a wild-type strain (NRRL 3357). To create strains with multiple oxygenase lesions, RNAi technology was employed using inverted repeat transgene of several oxygenases, as depicted in Supplementary Figure 2. Strain TJBS 5.1.60 carried the invert repeat transgene (IRT) of $p p o A$, and $p p o B$ in the $\triangle p p o D$ background was named IRT2 (Fig. 2D). Silencing of ppoC and lox genes in IRT2 created IRT4 ( $\triangle p p o D ; p p o A,-B$, and $-C$; and $l o x$ IRT), deficient in expression of all five oxygenases (Fig. 2E). As expected for IRT-containing strains, transcripts ( $p p o A$ and $p p o B$ in IRT2 [Fig. 2D] and $p p o A, p p o C$, and lox in IRT4 [Fig. 2E]) were degraded in these strains.

\section{Conidial and sclerotial density-dependent development is impaired in oxygenase mutants.}

Based on recent data where deletion of lox in A. flavus affected the sclerotia-to-conidial population shift (Horowitz et al. 2008), we predicted that ppo mutants would exhibit an altered density-dependent phenotype. The relative ability of the wild type, $\triangle p p o A, \Delta p p o C, \Delta l o x, \Delta p p o D$, IRT2, and IRT4 to form sclerotia and conidia was determined by inoculating $10^{2}$, $10^{4}$, and $10^{6}$ conidia on glucose minimal medium (GMM) plus $2 \%$ sorbitol plates which were placed in constant darkness at $29^{\circ} \mathrm{C}$ for 7 days. Cell density development patterns were skewed to various degrees among the oxygenase mutants compared with the wild type (Supplementary Fig. 3). Macroscopically, $\Delta p p o C, \Delta l o x$, IRT2, and IRT4 showed alterations in the 
sclerotia-to-conidia shift. In particular, IRT4 exhibited the most striking phenotype, with increased production of sclerotia and near absence of conidia at all populations. Conidia production decreased in $\triangle p p o C, \Delta l o x$, IRT2, and IRT4 strains at all population densities. IRT4 showed the most dramatic decrease in conidiation: 75,86 , and $97 \%$ reduction compared with the wild type at $10^{2}, 10^{4}$, and $10^{6}$ conidia/plate, respectively. In contrast to these mutants, the $\triangle p p o D$ strain showed an increase in conidiation compared with the wild type at $10^{6}$ conidia/plate (Fig. 3A). At low cell density $\left(10^{2}\right.$ conidia/plate), there was no difference in sclerotial production as measured by weight between any strains (Fig. 3B). At the intermediate population level $\left(10^{4}\right.$ conidia/plate $), \triangle p p o D$ showed a reduction in sclerotial production compared with all other mutants (Fig. 3B). At high cell densities, all strains containing a downregulated or deleted $p p o C$ or lox allele exhibited high sclerotial production. The loss of ppoA showed a minimal effect, with slightly more sclerotia formation at the highest cell density. In contrast, $\triangle p p o D$ was the only strain to produce less sclerotia than the wild type, which correlated with its increased conidial production (Fig. 3A and B). The most striking phenotype was the greatly increased production of sclerotia in IRT4 compared with the wild type by 114 and $532 \%$ at $10^{4}$ and $10^{6}$ cell densities, respectively (Fig. 3B).
Genes ppoC and lox cooperate in a synergistic manner to regulate cell density development.

The IRT2 strain (e.g., $p p o A, p p o B$, and $p p o D$ downregulated) did not show as much sclerotial increase as the IRT4 strain; therefore, it appeared that PpoC or Lox was most critical the overproduction of sclerotia by IRT4 (Fig. 3B). To assess the contribution of ppoC and lox individually to the IRT4 phenotype, $р р о C$ and $l o x$ were next inactivated independently in the IRT2 background to yield IRT2- $\Delta l o x$ and IRT2- $\Delta p p o C$. Sclerotia and conidial production were examined in 6-day-old cultures (Fig. 4A and B). Both the IRT2-Alox and IRT2- $\Delta p p o C$ strains produced an identical phenotype, intermediate to that of IRT2 and IRT4 (Fig. 4A).

\section{Secondary metabolite production, including AF, is regulated by cell density and disturbed in oxygenase mutants.}

To evaluate the effect of cell density on secondary metabolite biosynthesis, including AF (B1), cultures of A. flavus wild type at $10^{2}$ to $10^{7}$ conidia per plate were grown on GMM (to promote general secondary metabolite biosynthesis) (Fig. 5A, upper panel) or GMM plus $2 \%$ sorbitol (to promote AF biosynthesis) (Fig. 5A, lower panel) in constant darkness at $29^{\circ} \mathrm{C}$ for 6 and 3 days, respectively. Thin-layer chromatography (TLC) analysis

\section{A}

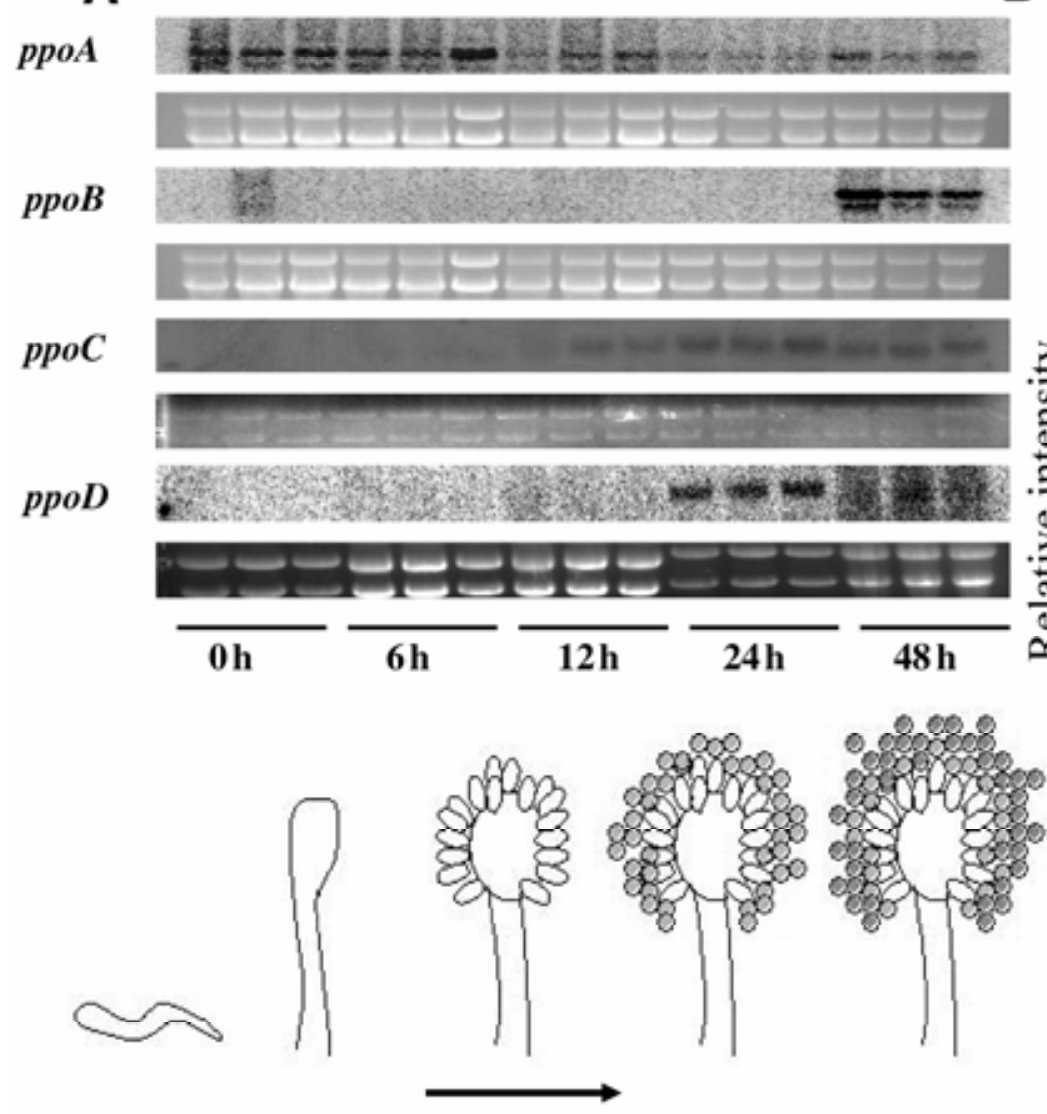

B
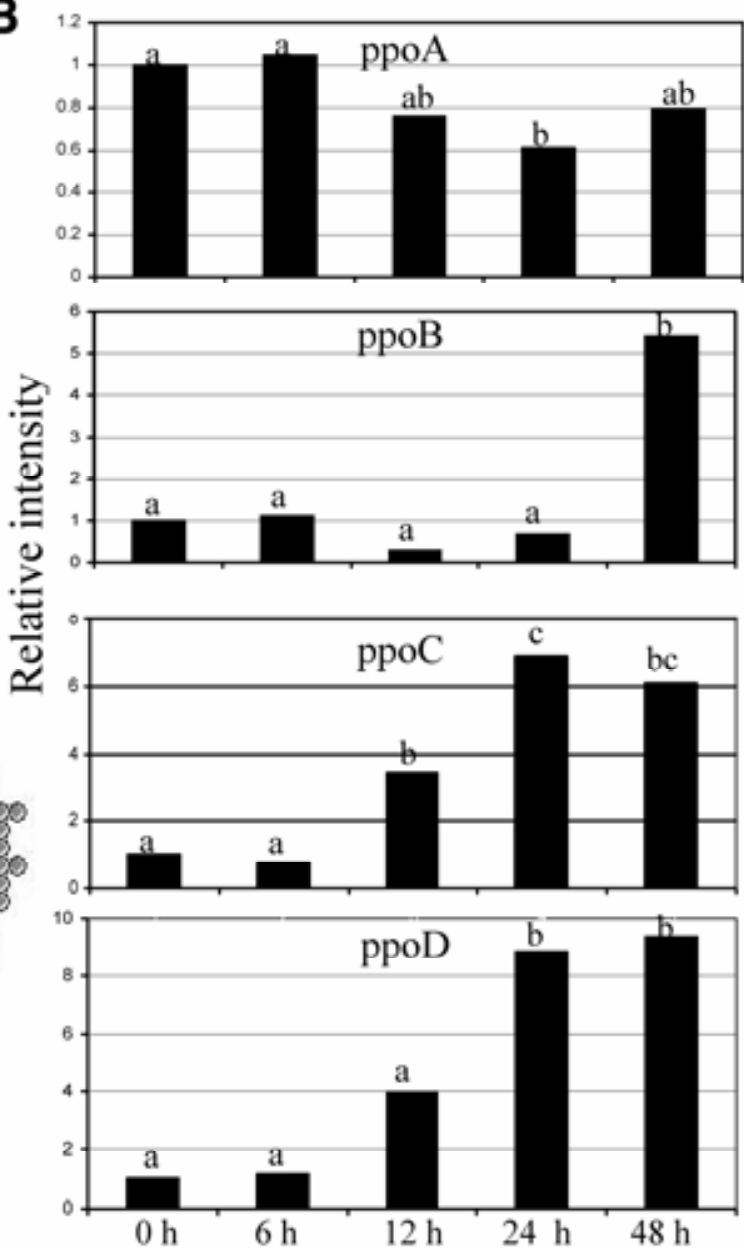

Fig. 1. Expression of ppo during conidiophore formation. A, Aspergillus flavus wild-type 3357 grown in liquid shaking culture (270 rpm, $\left.29^{\circ} \mathrm{C}\right)$ to competence $(22 \mathrm{~h})$ and transferred to filter paper on $2 \%$ sorbitol solid glucose minimal medium at $29^{\circ} \mathrm{C}$ under constant light. Total RNA was extracted from three replicates at $0,6,12,24$, and $48 \mathrm{~h}$ after the transfer. Northern blots were probed with internal fragments of each ppo. Below the Northern blots is a cartoon depiction of the predominant fungal tissue at each time point—competent tissue, vesicle, vesicle covered with phialides, young conidiophores, and mature conidiophores at each time point, respectively. Ethidium bromide-stained rRNA is shown as a loading control. B, Quantification of band intensity was performed by Image $\mathbf{J}$ software and expressed in terms of relative intensity compared with the standard. Statistical significance of quantification was assessed using Tukey-Kramer multiple comparison test $(P<0.05)$. 
showed that secondary metabolite production is density dependent in the wild type, where increasing cell density yields the lowest secondary metabolite production (Fig. 5A) - a pattern similar to that of sclerotial production. AF was detected only at the lowest cell density $\left(10^{2}\right.$ conidia/plate $)$ under the experimental conditions assayed (Fig. 5A, lower panel).

To study the role of oxygenases specifically on AF biosynthesis, we examined AF production in all mutants grown on GMM plus $2 \%$ sorbitol at three cell densities: $10^{2}, 10^{4}$, and $10^{6}$ (Fig. 5B). AF was detected in all strains at the lowest cell density. There was minimal AF production in $\Delta p p o C, \Delta l o x$, and IRT2 strains at the intermediate concentration of $10^{4}$ conidia/plate; however, the most striking finding was the high level of AF production in IRT4 at all population densities (Fig. 5B). To address the impact of $p p o C$ and lox individually on AF production in the IRT4 strain, we examined the IRT2- $\Delta p p o C$ and IRT2- $\Delta l o x$ strains for their ability to produce $\mathrm{AF}$ at high cell densities. The results demonstrate that reduction of both ppoC and lox is required to induce $\mathrm{AF}$ at high density (Fig. 5C).

\section{IRT 2 and IRT4 mutants are impaired in conidiation but not AF production on peanut and corn seed.}

To dissect the role of A. flavus oxygenases when the fungus is interacting with live seed, we examined the ability of

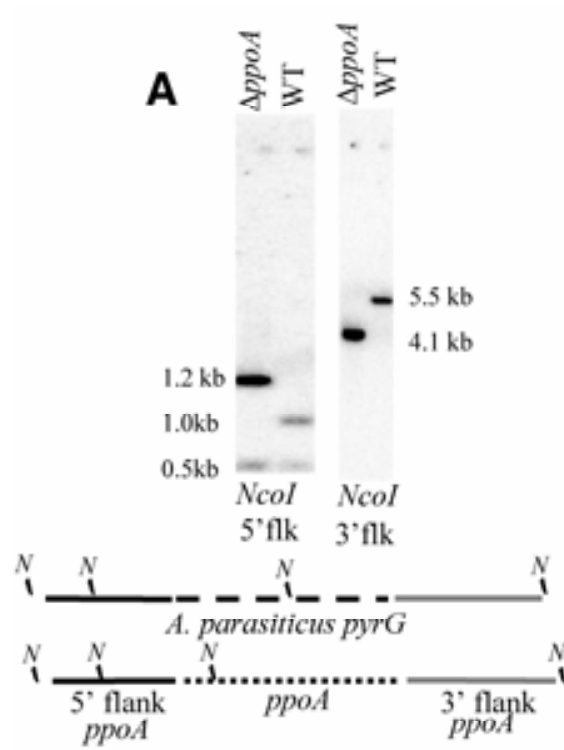

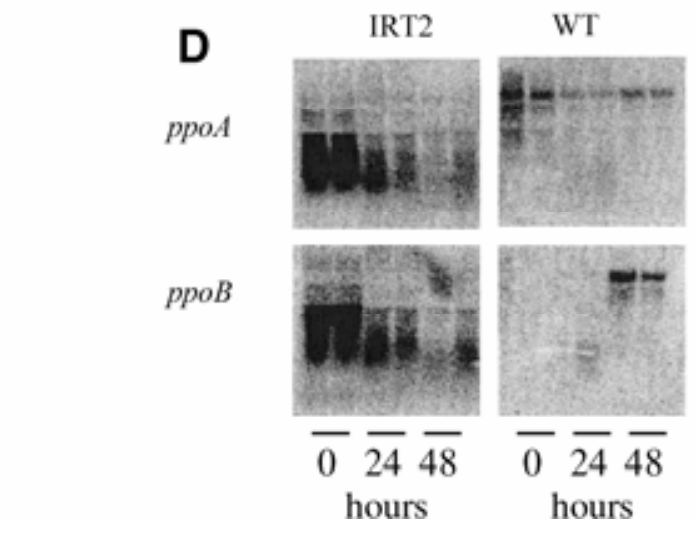
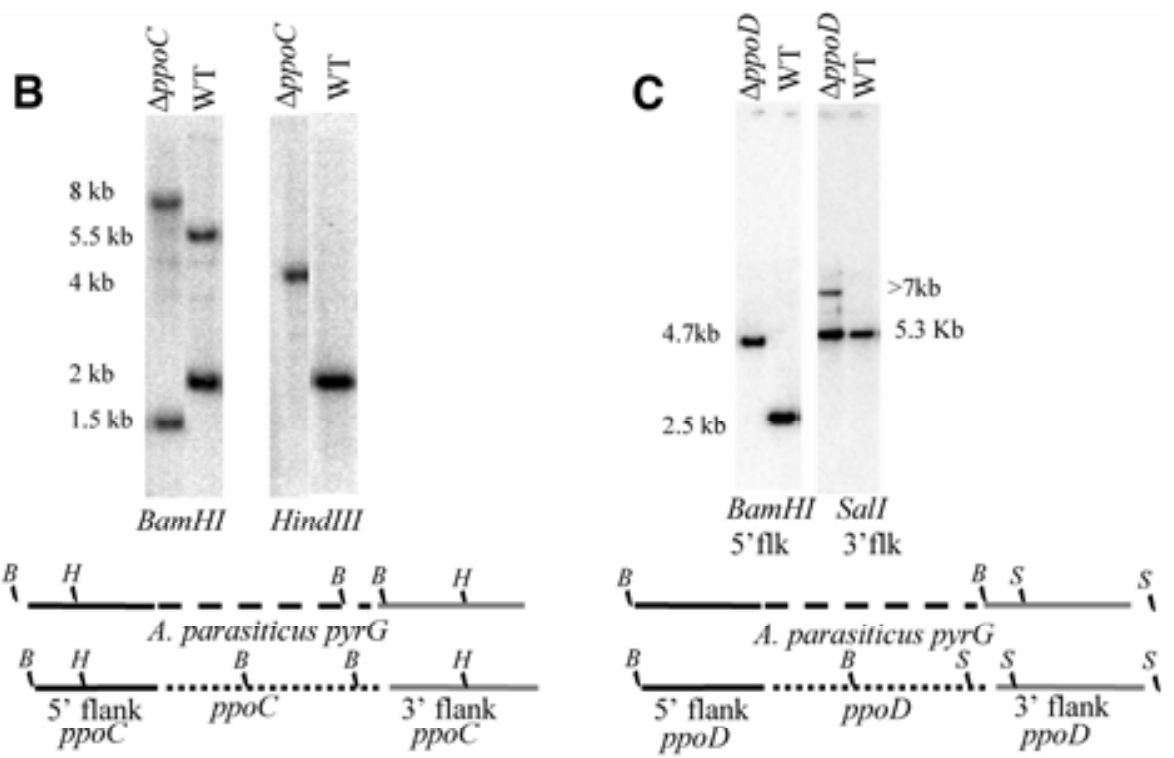

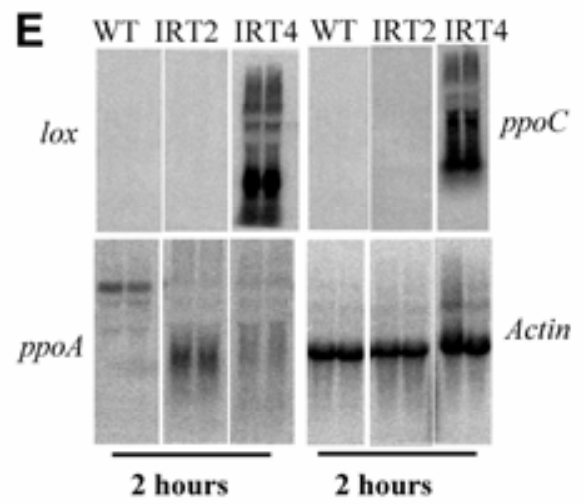

Fig. 2. Deletion and or silencing expression of ppo genes in Aspergillus flavus. A, Diagram of the $\mathbf{A}$, ppoA; $\mathbf{B}$, ppoC; and $\mathbf{C}$, ppoD coding sequence and how they were replaced with the A. parasiticus pyrG selectable marker by homologous recombination to generate the single deletion $\triangle p p o A, \triangle p p o C$, and $\triangle p p o D$ mutants, respectively. Southern analysis of wild-type NRRL 3357 strain (WT), $\triangle p p o A$ mutant TJBS3.1.19, $\Delta p p o C$ mutant TRAW2.2.15, and $\Delta p p o D$ TJBS4.1.85. To determine deletion of $p p o A, p p o C$, and $p p o D$, genomic DNAs were digested with NcoI, BamHI, HindIII, BamHI, and SalI as shown. PpoA: Expected hybridization band patterns by $N c o$ I digestion are a 0.5 - and 1.0-kb band for the WT strain and 0.5- and 1.2-kb bands for $\triangle p p o A$ strains (using the $3^{\prime}$ рроA flank as a probe). When the $5^{\prime}$ flank was used as a probe, the expected hybridization band patterns by $N c o$ I digestion are $5.5 \mathrm{~kb}$ for the WT strain and $4.1 \mathrm{~kb}$ for $\triangle p p o A$ mutants. PpoC: Expected hybridization band patterns for BamHI are 2- and 5.5-kb bands for the WT strain and 1.5- and 8-kb bands for $\triangle p p o C$ strains. Digestion with HindIII is a 2-kb band for the WT and a 4-kb for $\triangle p p o C$ when full-length $p p o C$ is used as a probe. PpoD: Expected hybridization band patterns with $B a m \mathrm{HI}$ is a $2.5-\mathrm{kb}$ band for the WT strain and a 4.7-kb for $\Delta p p o D$ strains with the $5^{\prime}$ ppoD flank as a probe. Digestion with HindIII gives a 5.3-kb band for the WT and $\geq 7 \mathrm{~kb}$ for $\triangle p p o D$ with the $3^{\prime} p p o D$ flank as a probe. D, Northern blots analysis to confirm if RNAi machinery successfully silenced both $p p o A$ and $p p o B$ genes in a $\triangle p p o D$ background is demonstrated. Expression of ppo in TJBS 5.1.60 (invert repeat transgene [IRT] strain IRT2) and the WT (NRRL 3357). In all, $10^{6}$ conidia from 5-day-old cultures were grown in liquid shaking culture $\left(270 \mathrm{rpm}, 29^{\circ} \mathrm{C}\right)$ to competence $(22 \mathrm{~h})$ and transferred to filter paper on $2 \%$ sorbitol solid glucose minimal medium (GMM) at $29^{\circ} \mathrm{C}$ under constant light. Total RNA was extracted from two replicates at 0,24 , and $48 \mathrm{~h}$ after the transfer. The presence of a smear for $p p o A$ and $p p o B$ in the IRT2 strain indicated a degraded transcript of the silenced genes as opposed to full-length transcripts in the WT. E, Northern blot analysis to study how lox, ppoA, and ppoC are silenced in IRT4 mutant. WT A. flavus, IRT2 (TJBS 5.1.60), and IRT4 (TSHB5.57) mutants were grown in liquid shaking culture $\left(270 \mathrm{rpm}, 29^{\circ} \mathrm{C}\right)$ to competence $(22 \mathrm{~h})$ and transferred to $2 \%$ sorbitol GMM liquid medium at $29^{\circ} \mathrm{C}$ under constant light, and total RNA was extracted from two replicates $2 \mathrm{~h}$ after the transfer. The presence of a smear for $p p o A$ (control), ppoC, and lox indicated a degraded transcript of the silenced genes as opposed to full-length transcripts in the WT. Actin probe was used as a control to demonstrate equal RNA loading. 
A. flavus oxygenase mutants to conidiate and produce AF in peanut (Fig. 6) and corn (Fig. 7) seed. IRT2 and IRT4 strains consistently produced less conidia than the wild type on both peanut and corn seed (Figs. 6 and 7). Conidial production was decreased $85 \%$ for both IRT2 and IRT4 compared with the wild type on peanut $(P \leq 0.05)$ and 90 to $97 \%$ on corn. However, despite the low levels of conidiation on the seed, IRT2 and IRT4 produced the most AF in the seed (Figs. 6B and 7B).

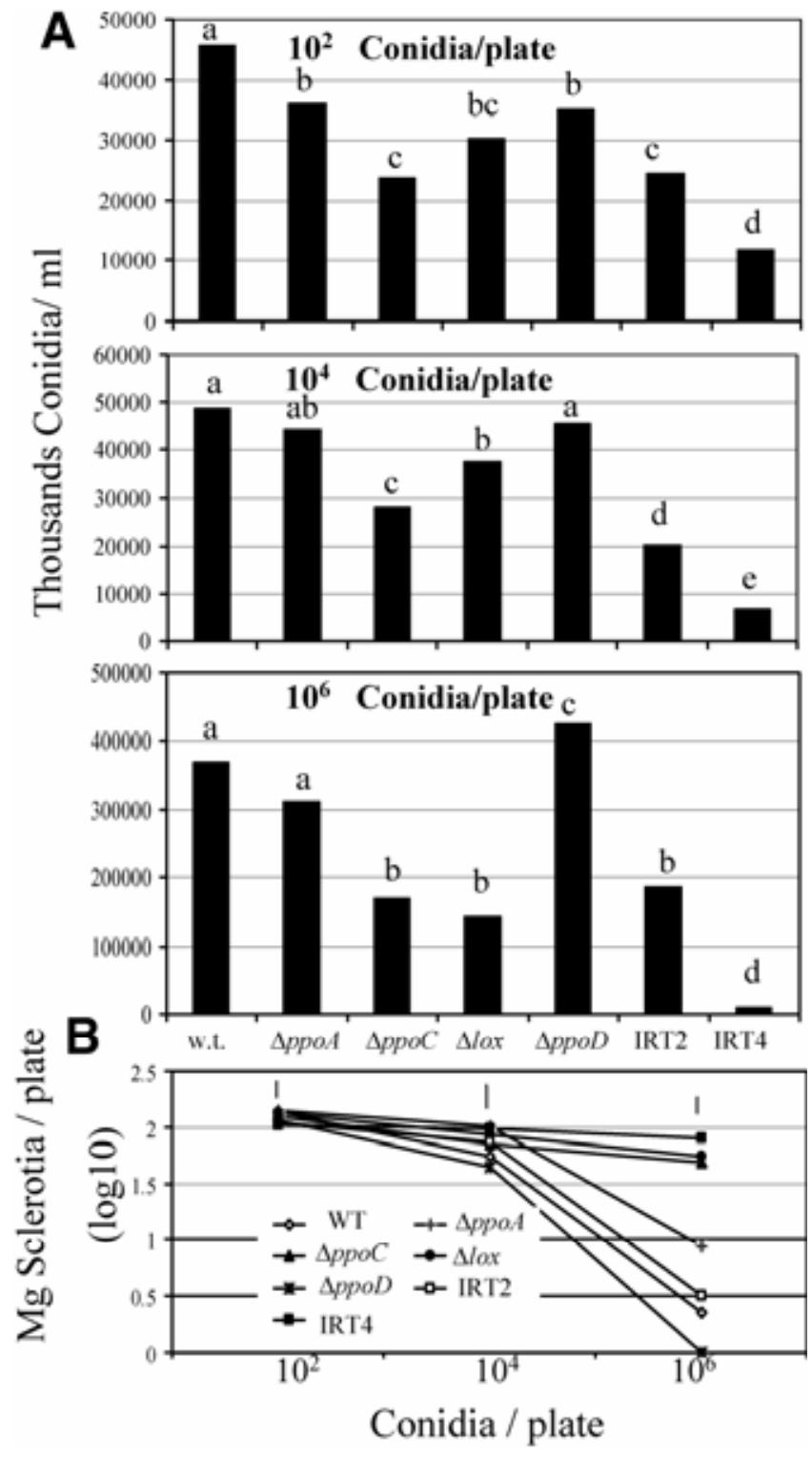

Fig. 3. Conidiation levels and sclerotia production are affected by oxygenase mutants. In all, $10^{2}, 10^{4}$, and $10^{6}$ conidia of wild-type strainNRRL 3357 (WT) and $\triangle p p o A, \triangle p p o C, \triangle p p o D, \Delta l o x$, invert repeat transgene (IRT)2, and IRT4 were overlaid on $10 \mathrm{ml}$ of glucose minimal medium plus $2 \%$ sorbitol and placed in constant darkness at $29^{\circ} \mathrm{C}$ for 3 days for assessment of conidiation levels and 7 days for assessment of sclerotia production. A, Conidial production was determined by counting under the microscope. Values are means of four replicates and tests were repeated three times in order to examine reproducibility. Spore suspensions were freshly prepared for each test. Letters (a, b, c, and d) indicate statistical differences $(P<$ $0.05)$ at different cell densities according to Tukey-Kramer multiple comparison test. B, Sclerotia production was determined gravimetrically (dry weight as milligrams per plate). Values are means of four replicates and tests were repeated three times in order to examine reproducibility. Spore suspensions were freshly prepared for each strain. Distances larger than the vertical bars between symbols at each cell density are significant $(P \leq$ 0.05 ) according to Tukey-Kramer multiple comparison test.

\section{DISCUSSION}

Density-dependent development in A. flavus is regulated through oxygenase expression.

In this study, we have characterized A. flavus fatty acid mutants deleted or downregulated for five oxygenase genes. Our findings provide evidence supporting an oxygenase signaling network that affects multiple developmental processes, including a quorum-like morphological shift from sclerotia to conidia formation, secondary metabolism, and host seed colonization. Our results further suggest that a pool of selected oxylipins are important for density-dependent morphology shifts in this fungus. The phenotypes of the individual oxygenase mutants in A. flavus suggested a possible redundancy in function. To address this possibility, two strains with multiple lesions were created. IRT2, a strain in which $p p o A$ and $p p o B$ were suppressed by RNAi in the $\triangle p p o D$ background, was reduced by almost $50 \%$ in conidiation compared with the wild type. Downregulation of all five oxygenases in A. flavus strain IRT4 resulted in overproduction of sclerotia and minimal conidiation through all cell densities These results support the hypothesis that single ppo deletion phenotypes may be masked or compensated by activity of other Ppos.

\section{Conserved oxylipin signaling among Aspergilli.}

In $A$. nidulans, independent inactivation of the three ppo genes resulted in alterations in the ratios of asexual to sexual spores produced. $\triangle p p o A$ and $\triangle p p o B$ strains were both increased in this ratio, whereas $\triangle p p o C$ strains displayed the opposite phenotype-increased in sexual spore and decreased in asexual spore production (Tsitsigiannis et al. 2004; 2005b). In $A$. fumigatus, the рроC mutant showed several abnormalities, including a decrease in spore production, aberrant conidiophore formation, and altered cell wall morphology (Dagenais et al. 2008; Garscha et al. 2007; Tsitsigiannis et al. 2005a). Although $A$. flavus ppoD deletion showed an increase in asexual reproduction (similar to $A$. nidulans ppoA and $p p o B$ mutants), the $\triangle p p o C$ and $\triangle l o x$ strains independently displayed a significant decrease in conidiation compared with the wild type at the high cell density (Fig. 3). Inactivation of PpoC-a highly conserved protein-shows some similarity among the three Aspergilli. In A. nidulans, $\triangle p p o C$ overproduces its resting stage, the cleistothecium, concomitant with decreased conidial production (Tsitsigiannis et al. 2004). In the asexual fungus A. fumigatus, $\triangle p p o C$ mutants also displayed decrease in conidiation (Dagenais et al. 2008). Results here show that A. flavus $\Delta p p o C$ mutants share a similar decrease in conidial production accompanied with overproduction of sclerotia at the high cell densities. This phenotype is very similar to deletion of the A. flavus lox gene (Fig. 3) (Horowitz et al. 2008). The PpoC protein has recently been found to produce $10 R$-hydroxy- $8 E, 12 Z$-octadecadienoic acid as its major oxylipin (Garscha et al. 2007). Possibly, this is a significant signaling molecule in Aspergillus spp. conidial development. A summary of the mutant phenotypes among the three Aspergilli species (Table 1) demonstrates some conserved oxylipin signaling patterns governing development.

\section{AF production is modulated by oxygenase activity.}

Lack of all three ppo genes in A. nidulans resulted in a strain unable to produce the mycotoxin sterigmatocystin (a precursor to AF) but upregulated in penicillin production (Tsitsigiannis et al. 2006). Here, we observed that, in the A. flavus IRT4 strain (reduced in expression of all five oxygenases), there was a dramatic increase in AF through all the cell densities (Fig. 5B). Our findings demonstrate that a combination of oxygenase activity is required for AF regulation. 
Oxylipin-mediated host pathogen cross talk.

Recent studies have uncovered a complex fungus-host interaction governed by oxylipins (Brodhagen et al. 2008; Gao et al. 2009). Here, we assessed the effect of deletion of oxylipin biosynthesis genes in A. flavus on disease seed colonization. Both IRT2 and IRT4 were significantly reduced in conidiation on peanut and corn seed compared with the wild type, yet contaminated the seed with high levels of AF (Figs. 6 and 7). These findings suggest that naturally occurring differences in oxygenase activity could explain some differences in $\mathrm{AF}$ production in field populations of $A$. flavus (Horn 2007) and also support reports where high levels of AF were found despite little evidence for fungal infection (Cotty and Mellon 2006).

Interestingly, conidiation and toxin production by $A$. flavus were increased during colonization of a maize lox3-4 mutant, as recently reported by Gao and associates (2009). Together with the data reported here, these observations suggest that at least certain oxylipins, directly or indirectly, act to limit AF production in situ. This interaction of fungal pathogen and the maize host is pathogen specific: in contrast to infection by Aspergillus spp., the maize lox3-4 mutant was more resistant to Fusarium verticillioides and Colletotrichum graminicola infections (Gao et al. 2007). The latter results may reflect many synergistic, opposing, or compensatory interactions among the fungal and seed oxylipin pool and serve to illustrate that potential oxylipin-based control strategies may need to be specified for each fungus-host interaction.

\section{Oxygenases as population-density-dependent determinants of fungal development.}

Quorum sensing is a density-dependent process that functions when a population is of certain size. Development and survival of any organism depends in large part on proper responses to the density of the surrounding population. In recent years, a quorum-sensing-like phenomena was uncovered in several yeast species. Each of these cases involves a morphological transition among dimorphic fungi, from a filamentous my-
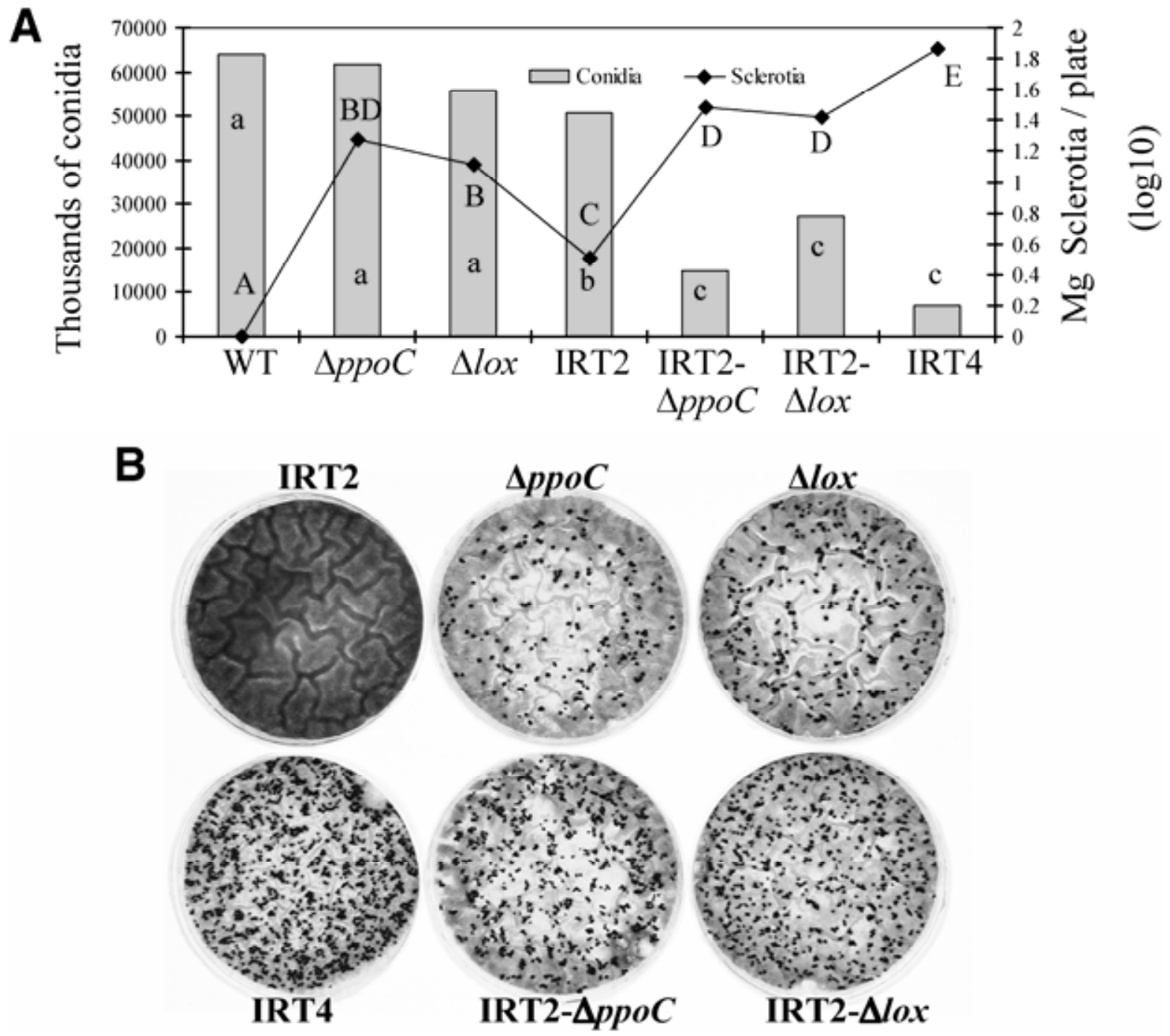

Fig. 4. ppoC and lox cooperate in a synergistic manner to regulate cell density development. A, To assess the contribution of $p p o C$ and lox individually to the invert repeat transgene (IRT)4 phenotype, wild-type strainNRRL 3357 (WT), single deletion of $\Delta l o x$ and $\Delta p p o C$, IRT2- $\triangle p p o C$, and IRT2- $\Delta l o x$ were compared with IRT2 and IRT4 strains. In all, $10^{6}$ conidia were overlaid on $10 \mathrm{ml}$ of glucose minimal medium plus $2 \%$ sorbitol and placed in constant darkness at $29^{\circ} \mathrm{C}$ for 6 days. Sclerotia production was determined gravimetrically (dry weight as milligrams per plate) and conidia by counting under the microscope. Values are means of four replicates and tests were repeated three times in order to examine reproducibility. Spore suspensions were freshly prepared for each test. Different letters (a, b, and c for conidia and A, B, and C for sclerotia) indicate statistical differences $(P<0.05)$ according to the Tukey-Kramer multiple comparison test. Data counted only for the high cell density, $10^{6}$ conidia/ml. B, Visual phenotype of sclerotia production at $10^{6}$ conidia/plate after 6 days of incubation; the additive effect of ppoC and lox deletion is demonstrated by increased sclerotia production in IRT4 compared with independent single deletion of ppoC and lox in IRT2 background. 
celial form to a yeast form, or vice versa (Sprague and Winans 2006). Investigations of quorum sensing in Candida albicans identified two quorum-sensing molecules: farnesol, which inhibits formation of germ tubes when cultures reach high density, and tyrosol, which promotes cell growth and the formation of germ tubes at low cell density (Chen and Fink 2006; Chen et al. 2004). Whole-genome microarray expression analysis suggest that these $C$. albicans quorum-sensing molecules may also play a role in signaling the transition to the stationary phase (Chen and Fink 2006). Recently, we reported that A. flavus not only demonstrates a quorum-sensing mechanism as illustrated in the sclerotia-to-conidia switch but also that Lox activity is important for this switch (Horowitz et al. 2008). Here, we show that the four Ppo proteins also play a critical role in this switch and that oxygenase activity also impacts an apparent density-dependent regulation of secondary metabolism, possibly related to sclerotial formation. It appears that the activity of PpoC and Lox oppose the role of PpoD in A. flavus development (Table 1), much like that seen in PpoC versus $\mathrm{PpoB}$ in $A$. nidulans. The accumulating data suggest that control of phase change and differentiation in A. flavus is modulated in part by oxylipins.

Quorum sensing can provide a means to respond effectively to changes in environmental opportunities and allow the fungus to response to nutrient availability and microbial competition. In A. flavus, a sentinel system regulating conidial and sclerotial shifts could be evolutionarily beneficial for the organism to help it respond to changing environmental conditions. The conidium is the dispersal spore expected to be produced at times of starvation, a condition that may arise as high populations compete for limited nutrients. Sclerotial production would be favorable in establishing a niche for the producing organism and deterring predators through production of toxic metabolites (Wicklow et al. 1982).

\section{MATERIALS AND METHODS}

\section{Fungal strains and growth conditions.}

A. flavus strains used and generated in this study are listed in Table 2. All strains used for physiological studies were prototrophic. All strains were grown at $29^{\circ} \mathrm{C}$ and maintained on GMM (Shimizu and Keller 2001) unless otherwise indicated. Cells were visualized by using an Olympus BX60F-3 microscope and an Olympus SZ-60 stereoscope.

\section{Assay for conidia and sclerotia density dependence.}

Conidial and sclerotial measurements followed the procedure outlined by Horowitz and associates (2008). Briefly, $10 \mathrm{ml}$ of $1.6 \% \mathrm{GMM}+2 \%$ sorbitol agar was overlaid with $3 \mathrm{ml}$ of $0.7 \%$ GMM agar $+2 \%$ sorbitol agar containing $10^{2}$ (low cell density), $10^{4}$ (intermediate cell density), or $10^{6}$ (high cell density) conidia/plate of each appropriate strain in a 60-by-15-mm petri plate. Plates were incubated at $29^{\circ} \mathrm{C}$ under continuous darkness for 7 days. To accurately visualize the sclerotia, plates were sprayed thoroughly with $70 \%$ ethanol in water to remove conidia and aerial mycelia. The exposed sclerotia then were collected and lyophilized, and sclerotium production was determined gravimetrically (dry weight per plate). Conidia were counted at 3 or 6 days. To accurately count conidia, three $1-\mathrm{cm}$ plugs were homogenized in $3 \mathrm{ml}$ of $0.01 \%$ Tween 80 water, diluted to $1 \times$ and counted with a hemacytometer at $\times 400$ magnification. For each treatment, conidia and sclerotia counts were performed using four replicates and tests were repeated three times in order to examine reproducibility. Spore suspensions were freshly prepared for each test.

\section{Secondary metabolites analysis.}

For total secondary metabolites analysis, $10^{2}$ to $10^{7}$ conidia per plate of the wild type were inoculated at each power of 10
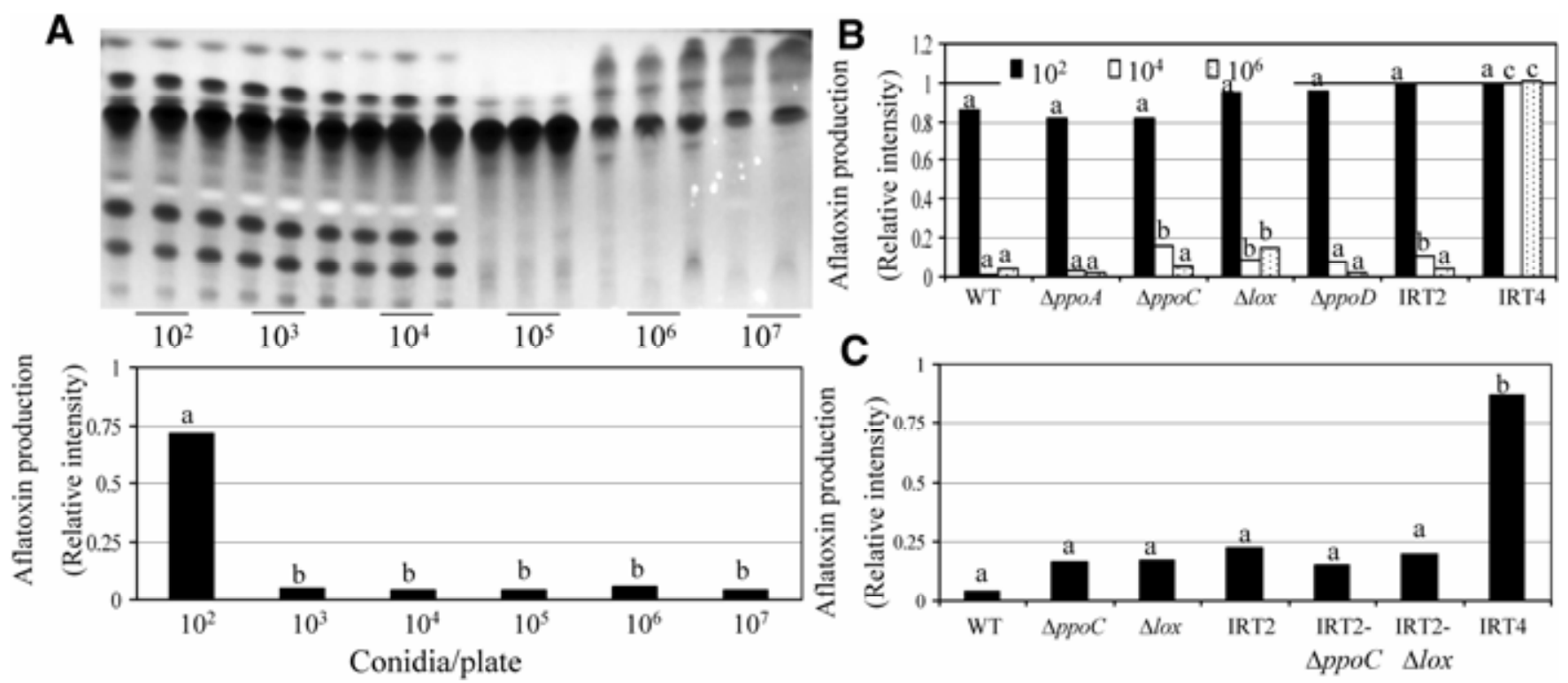

Fig. 5. Secondary metabolites and aflatoxin (AF) density dependence is affected by Aspergillus flavus oxygenases. A, Cell density regulates general secondary metabolites, including AF in A. flavus. In all, $10^{2}-10^{6}$ conidia of wild-type strainNRRL 3357 (WT) were overlaid stepwise to each power of 10 on $10 \mathrm{ml}$ of glucose minimal medium (GMM) alone for general secondary metabolites or plus $2 \%$ sorbitol for AF and placed in constant darkness at $29^{\circ} \mathrm{C}$ for 7 or 3 days, respectively. Metabolites were extracted from three 1-cm plugs in $1 \mathrm{ml}$ of chloroform and separated by thin-layer chromatography (TLC). B, To assess AF production in A. flavus oxygenase mutants, $10^{2}, 10^{4}$, and $10^{6}$ conidia from 3-day-old cultures were overlaid on $10 \mathrm{ml}$ of GMM plus $2 \%$ sorbitol in constant darkness. Silencing the expression of four A. flavus ppos and lox genes in $\triangle p p o D$ led to increased levels of AF production through all cell densities. C, Relative individual effect of ppoC and lox deletion on AF production in invert repeat transgene (IRT) 2 background. Reduction of both $p p o C$ and lox genes is required to induce $\mathrm{AF}$ at high cell densities, demonstrating the synergistic effect of both $p p o C$ and lox on AF production observed in IRT4 mutant. TLC analyses were repeated three times and quantified by image J software. Quantification of band intensity was expressed in terms of relative intensity compared with AF standard within the same TLC plate. Statistical significance of quantification was assessed using Tukey-Kramer multiple comparison test $(P<0.05)$. 
on GMM and placed in constant darkness at $29^{\circ} \mathrm{C}$ for 7 days. For AF analysis, GMM + 2\% sorbitol medium was overlaid with $3 \mathrm{ml}$ of a suspension of appropriate strains at $10^{2}, 10^{4}$, or $10^{6}$ conidia $/ \mathrm{ml}$ that were grown for 3 days at $29^{\circ} \mathrm{C}$. Three $1.5-\mathrm{cm}-$ diameter cores were harvested from the center of each plate and homogenized in $5 \mathrm{ml}$ of distilled water. Chloroform $(1 \mathrm{ml})$ was added and the mixture was vortexed for $1 \mathrm{~min}$, then centrifuged at $2000 \mathrm{rpm}$ for $10 \mathrm{~min}$. The lower organic layer was removed and evaporated. Residue was resuspended in $100 \mu \mathrm{l}$ of chloroform and $10 \mu \mathrm{l}$ of the suspension were spotted onto silica gel TLC plates (Whatman Ltd., Ken, England). Extracts were separated on the TLC plates using a benzene/methanol/acetic acid (80:15:5, vol/vol/vol) solvent system. Experiments were done in triplicate and repeated twice in order to examine reproducibility. AF standard was purchased from Sigma-Aldrich (St. Louis). AF was visualized using long-wave (366 nm) UV light and digital photographs were taken. Quantification of AF band intensity was performed by Image J software (version 1.24; developed at the National Institutes of Health) and expressed in terms of relative intensity compared with the AF standard. Statistical significance of quantification was assessed by mean comparisons according to the Tukey-Kramer multiple comparison test at $P \leq$ 0.05 . Mean values with different letters are significant.

Peanut and corn seed infection studies and AF analysis from seed.

Infection studies and AF analysis of peanut (Arachis hypogaea) cv. SunRunner and untreated maize seed (X516XW raw) obtained from Kaltenberg Seed Farm, Inc. (Waunakee, WI, U.S.A.) were performed as has been described previously (Burow et al. 2000; Tsitsigiannis and Keller 2006). For peanut infection, mature peanut seed were prepared by removing the brown exterior peanut layer (testa). The two cotyledons of each seed were separated and the embryo carefully removed without damaging any of the cotyledons tissue. Then, cotyledons were surface sterilized by placing them in a tea ball infuser and dipping them in a beaker containing $0.05 \%$ sodium hypochlorite in sterile water for $3 \mathrm{~min}$. The tea ball was transferred to a new beaker containing sterile distilled water for 30 s, followed by a 5 -s wash with $70 \%$ ethanol in a new beaker and one more 30-s wash with sterile distilled water while shaking the tea ball. The cotyledons were drained completely and placed in a petri dish until the time of infection. All steps were aseptically performed in a biosafety hood. Peanut cotyledons were inoculated with a $10^{5}$ spore $/ \mathrm{ml}$. Cotyledon treatments included water control (mock inoculation) and infection with fungal strains. For all treatments, 20 peanut cotyledons were immersed in $20 \mathrm{ml}$ of sterile distilled water (control) or sterile distilled water with fungal conidia in $50-\mathrm{ml}$ centrifuge tubes while shaking for $30 \mathrm{~min}$ in a rotary shaker at $50 \mathrm{rpm}$. Cotyledons were placed in petri dishes lined with three pieces of moist filter paper and a water reservoir to maintain high humidity. Cotyledons were incubated for 3 days at $29^{\circ} \mathrm{C}$ in dark conditions. For maize infection, untreated maize seed were inoculated similarly to peanut seed with the following differences. Seed were wounded by creating a small hole near the
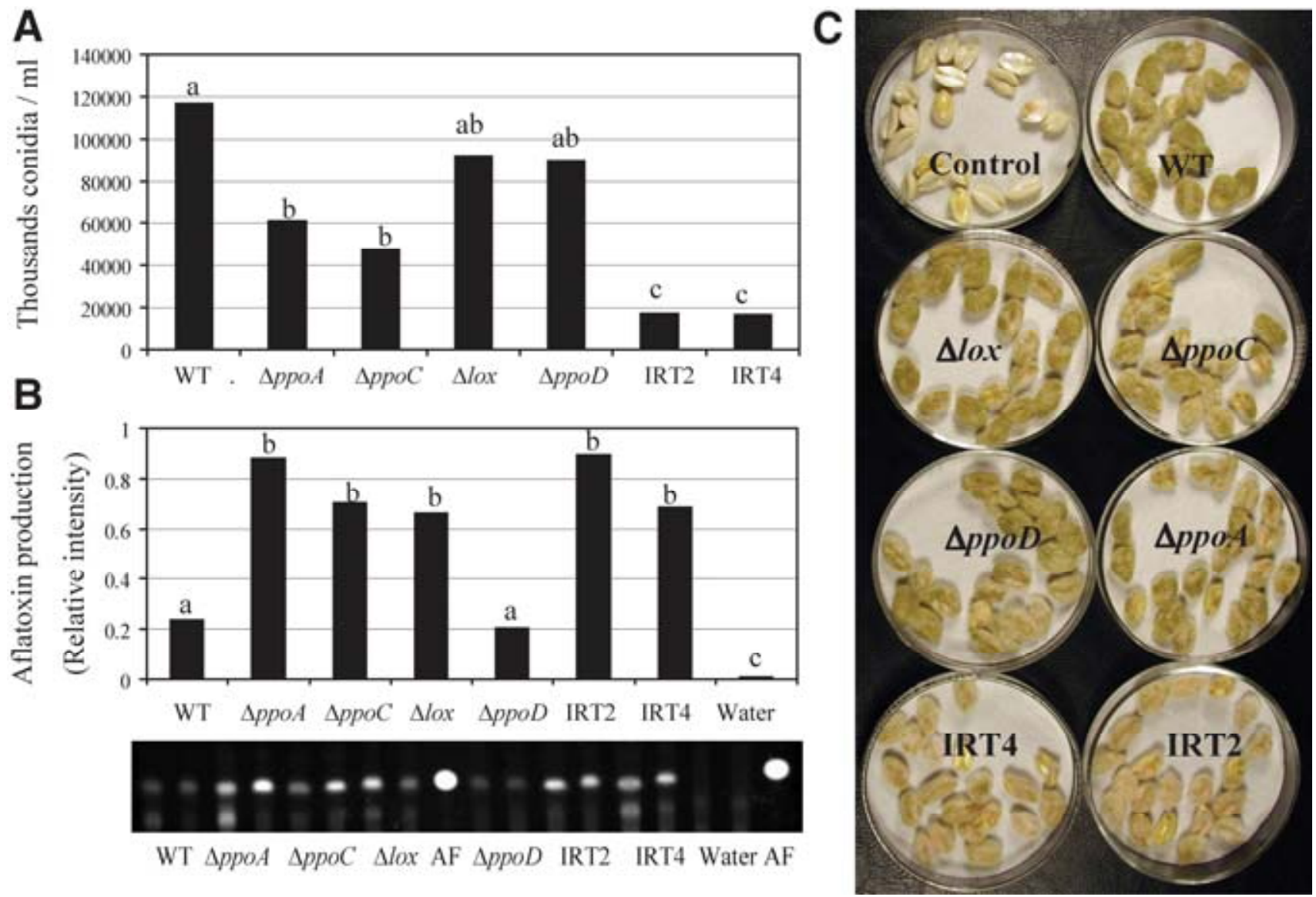

Fig. 6. A and B, Growth and aflatoxin (AF) production on peanut seed. Colonization of peanut seed as reflected by conidiation levels: Wild type NRRL 3357 (WT), $\triangle p p o A, \triangle p p o C, \triangle p p o D, \Delta l o x$, invert repeat transgene (IRT)2, and IRT4 were grown on peanut seed for 2 days at $29^{\circ} \mathrm{C}$ and $\mathbf{C}$, conidia were counted from four replicates containing 20 seeds each. Columns with the same letters are not significantly different at significance level of $P<0.05$. AF production in situ in peanut as examined by thin-layer chromatography (TLC) is shown in the lower panel. Water = mock inoculated with water and AF = AF B 1 standard. TLC analyses were quantified by image $\mathrm{J}$ software and band intensity was expressed in terms of relative intensity compared with AF standard within the same TLC plate. Columns with the same letters are not significantly different at significant level of $P<0.05$. 
embryo and then seed were immersed in $20 \mathrm{ml}$ of sterile distilled water (control) or sterile distilled water with fungal conidia at $10^{5}$ conidia/ $\mathrm{ml}$ in $50-\mathrm{ml}$ centrifuge tubes while shaking for $30 \mathrm{~min}$ in a rotary shaker at $50 \mathrm{rpm}$. Ten seeds per replicate were transferred to the humidity chamber and incubated in the dark at $29^{\circ} \mathrm{C}$ for 3 days. The filter paper was moistened daily and any germinating seed were removed and discarded. Analysis was performed on the same number of seed for each treatment and replication. All seed experiments were repeated three to four times. For AF extraction 3 days after infection, peanut cotyledons were collected in $50-\mathrm{ml}$ centrifuge tubes with the addition of $3 \mathrm{ml}$ of $0.01 \%$ Tween 80 ( $\mathrm{vol} / \mathrm{vol}$, water) and vortexed vigorously for $1 \mathrm{~min}$. One milliliter was removed from each sample for conidia counting prior to AF extraction. For extraction, $5 \mathrm{ml}$ of acetone was added to the samples followed by shaking for $10 \mathrm{~min}$ at $150 \mathrm{rpm}$. Samples were allowed to stand for an additional $10 \mathrm{~min}$ at room temperature, vortexed briefly, and centrifuged for $15 \mathrm{~min}$ at 2,000 rpm to collect the organic lower phase. Samples were then dried completely. The presence of abundant seed lipids in the samples hampered the clear observation of AF on TLC plates and a second extraction purification was carried out as follows. Samples were resuspended in $5 \mathrm{ml}$ of $0.1 \mathrm{M} \mathrm{NaCl}$ methanol/water (55:45) and $2.5 \mathrm{ml}$ of hexane and vortexed vigorously at high speed for $1 \mathrm{~min}$. Samples were centrifuged at 2,000 rpm for 5 min. The hexane layer was collected and the fatty acid inter- phase layer was discarded. The remaining aqueous phase was washed an additional time with $2.5 \mathrm{ml}$ of hexane as described above. The hexane extracts were combined, allowed to dry, and then resuspended in $500 \mu \mathrm{l}$ of chloroform before $10 \mu \mathrm{l}$ of each extract was separated on a silica gel TLC plate using a chloroform/acetone (95:5, vol/vol) solvent system. Maize seed were extracted similarly but without the needed extra hexane extraction for peanut seed. Quantification of band intensity was performed by Image J software (version 1.24, developed at the National Institutes of Health) and expressed in terms of relative intensity compared with AF standard. Statistical significance of quantification was assessed by mean comparisons according to the Tukey-Kramer multiple comparison test at $P \leq$ 0.05 . Mean values with different letters are significant.

\section{Nucleic acid analysis.}

The extraction of DNA from fungi, restriction enzyme digestion, gel electrophoresis, blotting, hybridization, and probe preparation were performed by standard methods (Sambrook et al. 1989). To examine $p p o A, p p o B, p p o C$, and $p p o D$ gene expression, wild-type NRRL 3357 was grown to competency by inoculating $500 \mathrm{ml}$ of liquid GMM with $10^{6}$ conidia/ml and shaking at $250 \mathrm{rpm}$ for $22 \mathrm{~h}$. The competent tissue was divided into 50-ml aliquots, filtered onto 7-cm Whatman coarse filter paper, and grown on $2 \%$ sorbitol GMM in continuous white light at $29^{\circ} \mathrm{C}$ for $0,6,12,24$, or $48 \mathrm{~h}$. Total RNA was extracted
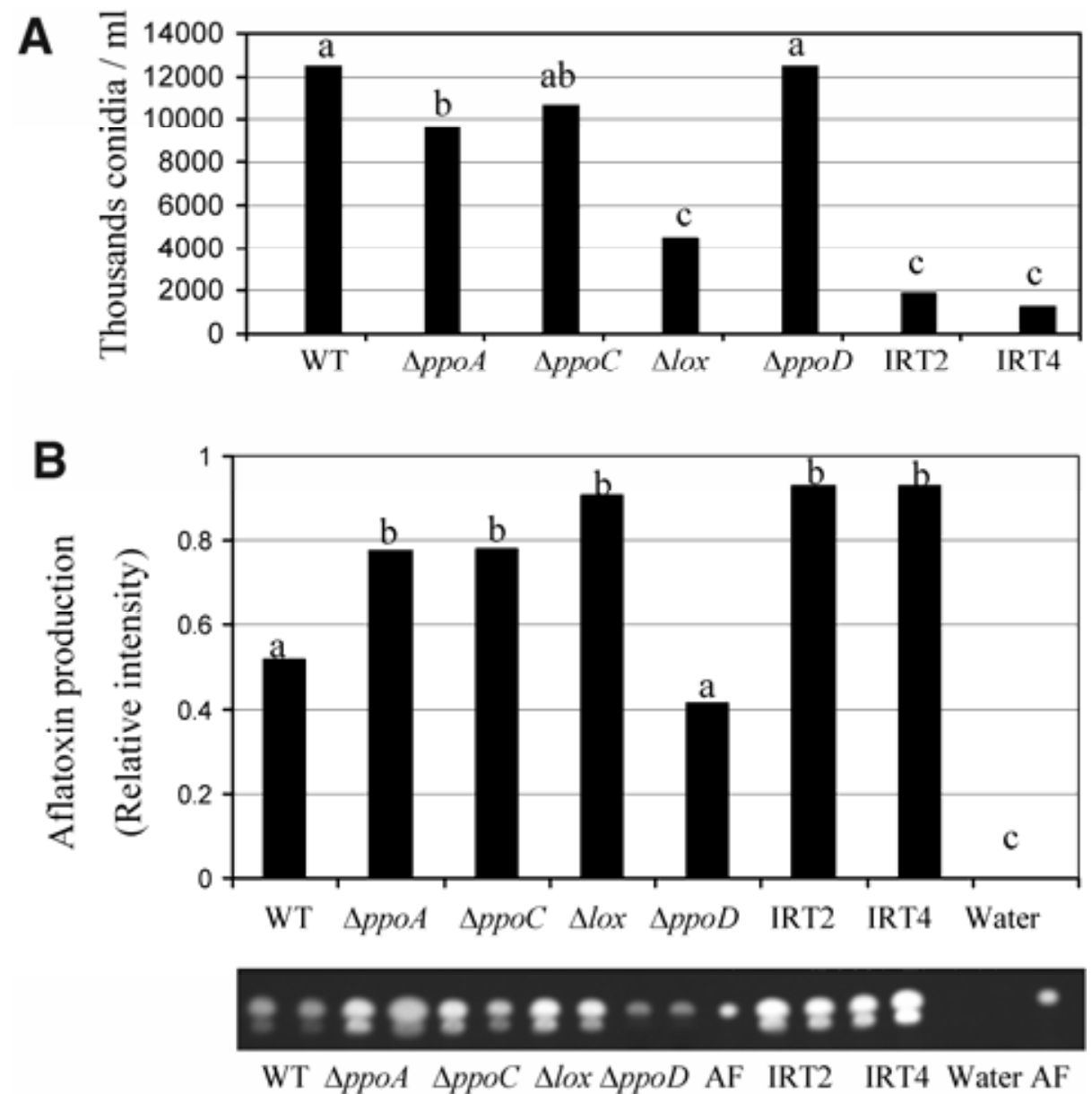

Fig. 7. Growth and aflatoxin production on corn seed. A, Colonization of corn seed as reflected by conidiation levels: Wild type NRRL 3357 (WT), $\triangle p p o A$, $\triangle p p o C, \triangle p p o D, \Delta l o x$, invert repeat transgene (IRT)2, and IRT4 were grown on corn seed for 3 days at $29^{\circ} \mathrm{C}$ and conidia were counted from four replicates containing 10 seeds each. Columns with the same letters are not significantly different at significance level of $P<0.05$. B, AF production in situ in corn as examined by thin-layer chromatography (TLC) is shown in the lower panel. Water = mock inoculated with water and AF = AF B1 standard. TLC analyses were quantified by image $\mathrm{J}$ software and band intensity was expressed in terms of relative intensity compared with AF standard within the same TLC plate. Columns with the same letters are not significantly different at significant level of $P<0.05$. 
from lyophilized mycelia using Trizol reagent (Invitrogen, Carlsbad, CA, U.S.A.). Approximately $20 \mu \mathrm{g}$ total RNA was run in a $1.2 \%$ agarose, $1.5 \%$ formaldehyde gel and then transferred to Hybond-XL membranes (Amersham Bioscience, Piscataway, NJ, U.S.A.) by capillary action. RNA transcript analysis was performed using random primer radiolabeled DNA fragments (Sambrook et al. 1989) derived from genomic polymerase chain reaction (PCR) products. The expression studies were performed with ppo-specific DNA probes which were generated from genomic DNA using the following primer combinations for each ppo gene: for $p p o A$, a 1.17-kb PCR fragment was amplified with $p p o A$ Int Fwd and $p p o A$ Int rev; for $p p o B$, a $0.85-\mathrm{kb}$ PCR fragment was amplified with $p p o B$ Int Fwd and $p p o B$ Int rev; for $p p o C$, a 1.4-kb PCR fragment was amplified with $p p o C$ Int Fwd and $p p o C$ Int rev; and, for $p p o D$, a 1.3-kb PCR fragment was amplified with $p p o D$ Int Fwd and $p p o D$ Int rev. Primer sequences are listed in Table 3. Detection of signals was carried out with a Phosphorimager-SI (Molecular Dynamics, Sunnyvale, CA, U.S.A.). The resulting expression analysis was measured by means of the public National Institutes of Health image processing program Image
$\mathrm{J}$ and are presented as relative intensity compared with time 0 presenting the vegetative growth stage. Two separate repetitions of this experiment yielded similar results. Nucleotide sequences were analyzed and compared using Sequencher (Gene Codes) and ClustalW programs (Chenna et al. 2003).

\section{Replacement and IRT constructs of Aspergillus flavus ppo and lox genes.}

The ppo genes were identified by nucleotide (blastn) search of the genomic scaffold of $A$. flavus based on the amino acid sequence of $A$. nidulans, $A$. fumigatus, and A. oryzae Ppo homologs. The ppo deletion constructs, including the $A$. parasiticus pyrG marker gene and the ppo flanking sequences, were constructed using the following methodology. Oligonucleotides used in this study (Table 3 ) were designed based on predicted open reading frames (ORF) and intergenic regions in these contigs. These primers were used in appropriate pairs to amplify 1.0 - to $1.2-\mathrm{kb}$ fragments of flanking regions by PCR using A. flavus genomic DNA as a template. The ppo flankingregion PCR products were first subcloned into PCR2.1 TOPO (Invitrogen) and then into pJW24 (Calvo et al. 2004) which

Table 1. Synopsis of oxygenase mutant phenotypes of Aspergillus nidulans, A. fumigatus, and A. flavus ${ }^{\mathrm{a}}$

\begin{tabular}{|c|c|c|c|c|c|c|}
\hline Gene & Conidiation $^{b}$ & $\begin{array}{l}\text { Resting } \\
\text { structure }^{c}\end{array}$ & $\begin{array}{l}\text { Secondary } \\
\text { metabolism }\end{array}$ & Pathogenicity & Other & Source \\
\hline \multicolumn{7}{|l|}{ A. nidulans } \\
\hline$\triangle p p o A$ & Slightly increased & Reduced & Slightly reduced & NA & $\ldots$ & $\begin{array}{l}\text { Tsitsigiannis et al. } \\
\text { 2004, 2005b, } 2006 .\end{array}$ \\
\hline$\triangle p p o B$ & Increased & Reduced & Increased & $\begin{array}{l}\text { Increased conidiation } \\
\text { and maceration }\end{array}$ & $\begin{array}{l}\text { Increased lipase } \\
\text { activity }\end{array}$ & $\begin{array}{l}\text { Tsitsigiannis et al. } \\
2005 b, 2006 \text {. }\end{array}$ \\
\hline$\Delta p p o C$ & Reduced & Increased & Increased & NA & $\ldots$ & $\begin{array}{l}\text { Tsitsigiannis et al. } \\
\text { 2004, 2005b, } 2006 .\end{array}$ \\
\hline АрроА, АрроВ, АрроС & Reduced & Increased & $\begin{array}{l}\text { ST reduced, } \\
\text { Penicillin increased }\end{array}$ & $\begin{array}{l}\text { Reduced sporulation } \\
\text { and ST }\end{array}$ & $\begin{array}{l}\text { Reduced lipase } \\
\text { activity }\end{array}$ & $\begin{array}{l}\text { Tsitsigiannis et al. } \\
2005 \mathrm{~b}, 2006 .\end{array}$ \\
\hline \multicolumn{7}{|l|}{ A. fumigatus } \\
\hline$\triangle p p o C$ & Reduced & None & NT & Reduced & $\begin{array}{l}\text { Aberrant conidial } \\
\text { attachment }\end{array}$ & Dagenais et al. 2008. \\
\hline рроА, рроВ рроС IRT & NA & None & NA & $\begin{array}{l}\text { Increased } \\
\text { (Murine model) }\end{array}$ & $\ldots$ & $\begin{array}{l}\text { Tsitsigiannis et al. } \\
2005 \mathrm{a}\end{array}$ \\
\hline \multicolumn{7}{|l|}{ A. flavus } \\
\hline АрроА & NA for most part & NA for most part & NA & NA & $\ldots$ & This study \\
\hline$\triangle p p o C$ & Reduced & Increased & NA & NA & $\ldots$ & This study \\
\hline$\triangle p p o D$ & Increased & Reduced & NA & NA & $\ldots$ & This study \\
\hline lox & Reduced & Increased & NA & NA & $\ldots$ & Horowitz et al. 2008 \\
\hline IRT2 & Reduced & NA & NA & Reduced conidiation & $\begin{array}{l}\text { Reduced lipase } \\
\text { activity }\end{array}$ & This study \\
\hline IRT2- $\Delta p p o C$ & Reduced & Increased & NA & NT & $\ldots$ & This study \\
\hline IRT2-Alox & Reduced & Increased & NA & NT & $\ldots$ & This study \\
\hline IRT4 & Reduced & Increased & Increased & Reduced conidiation & $\ldots$ & This study \\
\hline
\end{tabular}

${ }^{a} \mathrm{NA}=$ no alteration and $\mathrm{NT}=$ not tested.

${ }^{\mathrm{b}}$ Conidiation assessed in fungal tissue culture.

${ }^{\mathrm{c}}$ Resting structure referred to the A. nidulans sexual stage cleistothecia or sclerotia in A. flavus. None $=A$. fumigatus has no resting structure.

${ }^{\mathrm{d}}$ Secondary metabolism refers to Sterigmatocystin (ST) and Penicillin among A. nidulans strains, gliotoxin for A. fumigatus, and aflatoxin (AF) for A. flavus strains.

Table 2. Aspergillus flavus strains used in this study

\begin{tabular}{|c|c|c|}
\hline Strain $^{a}$ & Genotype & Source \\
\hline NRRL 3357 & Wild type & He et al. 2007 \\
\hline NRRL 3357.5 & pyrG & He et al. 2007 \\
\hline TSHB2.39 & AAflox:pyrG; pyrG & Horowitz et al. 2008 \\
\hline TSHB3.1.19 & АppoA:pyrG; pyrG- & This study \\
\hline TRAW2.2.15 & $\Delta p p o C: p y r G ; p y r G-$ & This study \\
\hline TJBS4.1.85 & LppoD:pyrG;pyrG & This study \\
\hline TJBS5.1.60 & АppoD:pyrG;niaD-:ppoA, pроB IRT:niaD & This study \\
\hline TSHB5.57 & АppoD:pyrG;niaD:ppoA, pроB IRT:niaDppoC, lox IRT:phleomycin & This study \\
\hline TSHB6.3 & sppoD:pyrG;niaD:ppoA, ppoB IRT:niaDAlox:pyrG- & This study \\
\hline TSHB7.1 & АppoD:pyrG;niaD:ppoA, ppoB IRT:niaD $\Delta p p o C: p y r G-$ & This study \\
\hline MJBS 4.0.50 & niaD-; ppoD:pyrG; pyrG- & This study \\
\hline MTJBS5.1.60A & PyrG-,PyrG- $\Delta p p o D: p y r G ; n i a D: p p o A$, pроB IRT:niaD & This study \\
\hline
\end{tabular}

${ }^{a}$ Strains starting with $\mathrm{T}$ were original transformants and those starting with $\mathrm{M}$ resulted from chemical-induced selection. 
contains the $A$. parasiticus pyrG marker gene surrounded by multiple cloning sites. The $5^{\prime}$ and $3^{\prime}$ flanking regions of $p p o A$ were amplified using the primers $p p o A$ fllk Fwd (SalI), ppoA 5flk Rev (EcoRI) and ppoA 3flk Fwd (BamHI), ppoA 3flk Rev $(X b a \mathrm{I})$, respectively. Each primer contains the engineered restriction site indicated. The 5' flank was inserted into pJW24 first with $S a l$ I and EcoRI to create pJBS3.0, followed by insertion of the $3^{\prime}$ untranslated region using BamHI and $\mathrm{XbaI}$ to complete pJBS3.1. To create the $p p o D$ disruption vector, 5' and $3^{\prime}$ flanking regions of the gene were amplified using the primers ppoD 5flk Fwd (SalI), ppoD 5flk Rev (EcoRI) and ppoD 3flk Fwd (BamHI), ppoB 3flk Rev (SpeI), respectively. Each primer contains the engineered restriction site indicated. Initially, the 5' flank was inserted into pJW24 using SalI and EcoRI to create pJBS4.0. The 3' flank was inserted by BamHI and SpeI digest to create the final disruption vector, pJBS4.1. To create the $p p o C$ disruption vector, flanking regions of the gene were amplified using the primers $p p o C 5$ flk Fwd $(S p h \mathrm{I})$ and ppoC 5flk Rev (SalI) for the 5' flanking region. The 3' flanking region of the gene was amplified by ppoC 3flk Fwd $(B a m \mathrm{HI})$ and ppoC 3flk Rev (EcoRI). Initially, the 5' flanking region was inserted by SphI and SalI into PUC18 vector (GenScript Corp., Piscataway, NJ, U.S.A.) containing a $2.8-\mathrm{kb}$ pyrG cassette to create pRAW2.1. Then, the 3' flank was inserted by BamHI and EcoRI digest to create the final disruption vector, TRAW2.2.

\section{niaD and pyrG auxotroph selection procedure.}

To facilitate the creation of strains of A. flavus with multiple ppo deletions, it was necessary to create an auxotrophic marker in one of the ppo deletion strains. One isolate of $\triangle p p o D$
(TJBS4.1.85) was mutated to nitrate reductase auxotrophy following a modified form of the method outlined by Cove (1976). Briefly, $10^{5}$ conidia of TJBS4.1.85 were inoculated in $500 \mu \mathrm{l}$ of sterile $.01 \%$ Tween water evenly spread on 25 solid plates of fresh $\mathrm{KCLO}_{3}$ medium. The cultures were incubated for 3 days at $29^{\circ} \mathrm{C}$ under constant light. Isolates with nonfunctional niaD are resistant to high concentration of $\mathrm{KClO}_{3}$. Resistant isolates were transferred to GMM and ammonium GMM (GMM with $5 \mathrm{mM}$ ammonium tartrate and no nitrate salts). Isolates that showed normal growth on $5 \mathrm{mM}$ ammonium tartrate GMM and poor growth on GMM were screened on nitrogen test media. Ten of these isolates were screened for positive growth on four different nitrogen sources: $5 \mathrm{mM}$ ammonium tartrate, $10 \mathrm{mM}$ sodium nitrate, $10 \mathrm{mM}$ sodium nitrite, and $100 \mathrm{mM}$ hypoxanthine. Isolates with a nonfunctional niaD $\left(\right.$ nia $\left.D^{-}\right)$grew normally on each of the four nitrogen sources except sodium nitrate, where they were visibly reduced in diameter growth and conidiation. Of the 10 isolates screened on the nitrogen sources, 3 were niaD $D^{-}$mutants. None of the three niaD ${ }^{-}$mutants had apparent growth defects compared with NRRL 3357 when growing on non-nitrate media. One nia ${ }^{-}$mutant, MJBS 4.0.50, was selected for RNAi silencing of $p p o A$ and $p p o B$ by transformation with pJBS5.1. The resulting $\triangle p p o D$, IRT $p p o A, p p o B$ triple mutant was designated TJBS5.1.60 and commonly referred to as IRT2 in this article. Later, IRT2 was used to generate a uracil auxotroph (a pyrG mutant) designated MTJBS5.1.60A, following the method outlined by $\mathrm{He}$ and associates (2007). MTJBS5.1.60A was used to create two more strains, IRT2- $\triangle p p o C$ and IRT2- $\Delta$ lox, using the TRAW2.2 construct for $p p o C$ deletion or pSHB2 construct for lox deletion (Horowitz et al. 2008), both carrying the

Table 3. Oligonucleotides used in this study

\begin{tabular}{|c|c|}
\hline Name & Sequence $5^{\prime}-3^{\prime}$ \\
\hline ppoA 5flk Fwd & CCTCAGCCGTCGACTCGTATAC \\
\hline PpoA 5flk Rev & CCTGAATTCATTGTTAGACGTGCTCG \\
\hline PpoA 3flk Fwd & CCGCTCGGATTCTGACTGTAAGTTTA \\
\hline PpoA 3flk Rev & TCCAACAGTAATCTAGAAAAGTCGAG \\
\hline PpoA Int Fwd & CCCCGGTTTCGTATGTTGTTTGATA \\
\hline PpoA Int Rev & TCCTCAACGCTGTCCTCAAAGATTT \\
\hline PpoA Fusion Fwd & GAAATGAAGCTTGGATGGCGCGCCGAAGCAGCCGCTCAGGCCAC \\
\hline PpoA Fusion Rev & GGTTGCCCAAAGCCGTGGGAACTGGTGATCATATAGTCGCTTGAGC \\
\hline PpoB 5flk Fwd & CTACCGAAGTCGACCCCAGAGATCCTCGCGC \\
\hline PpoB 5lk Rev & CAGGGCAAGGTTGGGTTATTGTAGAATTCGTCAG \\
\hline PpoB 3flk Fwd & GTAGATGGATCCAAATCATTCAGACTGCGCC \\
\hline PpoB 3flk Rev & TACTTCCAGCATTTACGTAGAATCCACGGA \\
\hline PpoB Int Fwd & TACGATAATGACCTTTTCCAGACG \\
\hline PpoB Int Rev & TATATTCCTTCTGTTCCGGTGTCA \\
\hline PpoB Fusion Fwd & CGAGTTCGCTGATATACTAGTGGTCAAGGGTGCCGAAACCCGTTGG \\
\hline PpoB Fusion Rev & ACACTGCCCGGGAGACGGTGGATCCTGGAGGCGCGCCAACA \\
\hline PpoC 5flk Fwd & GAAACAAAGCAAAATGGCATGCAAAATGGCGCTC \\
\hline ppoC 5flk Rev & GTTCCCCTCGCAGCATCGAACAGG \\
\hline ppoC 3flk Fwd & GGCTCCCTTAATGGATCCCGCAAG \\
\hline ppoC 3flk Rev & GGTAAAGACTCCGCCGCGCGCGAATTCTCCTTGG \\
\hline ppoD 5flk Fwd & GACTTGTCGACCTTCTCCCATTTTC \\
\hline PpoD 5flk Rev & CTTGAATTCGGGAACGTTTCTCGCAT \\
\hline ppoD 3flk Fwd & GCGTGTAAGGATCCCGATGCTGTC \\
\hline PpoD 3flk Rev & TATTTATTGTACTAGTCATCTGATCTTG \\
\hline ppoD Int Fwd & TCCGTGTTATTGAGATTCTGGGTA \\
\hline PpoD Int Rev & AACGATTCCCTTTCCAGATTTCTC \\
\hline NiaD Fwd & TGCACCTGTTATTATTAGTCTTACT \\
\hline NiaD Rev & AGAGAAGCGCCTACATGTGTATT \\
\hline Lox Int Fwd & AGGCCCGTATGAGCAGTTGAGT \\
\hline Lox Int Rev & GTTACCTTTACGGCTCCCCTCT \\
\hline Actin Int Fwd & ACAGTCCAAGCGTGGTATCC \\
\hline Actin Int Rev & GAAGCGGTCTGAATCTCCTG \\
\hline ppoC Fusion Fwd & AACTCTACCCTGGTATTGTTGCTGAGG \\
\hline PpoC Fusion Rev & GTCTAAGGGCAGGTGGTCGTTACTGGGATGGAGACGCTGGTAGAACCTGAGAATAGGTTA \\
\hline Lox Fusion For & GTCTCCATCCCAGTAACGACCACCTGCCCTTAG \\
\hline Lox Fusion For & TCTCAACGAACACGCTGGTGAAGTCTTCAATGG \\
\hline
\end{tabular}


pyrG as selectable marker. The resulting strains, TSHB6.3 and TSHB7.1, were designated IRT2- $\Delta l o x$ and IRT2- $\Delta p p o C$ through the article.

\section{Plasmids constructions}

to silence the $p p o A$, ppoB, ppoC, and lox expression.

pTMH44.2, containing the A. nidulans gpdA promoter and $\operatorname{trp} C$ terminator separated by approximately $280 \mathrm{bp}$ of green fluorescent protein $(g f p)$ with small multiple cloning sites upstream and downstream of $g f p$ (McDonald et al. 2005), was utilized to construct both $p p o A:: p p o B$ RNAi and $p p o C:: l o x$ RNAi vectors. Gene fragments from $p p o A$ and $p p o B$, approximately $500 \mathrm{bp}$ in length with no predicted introns, were amplified using the following primers (Table 3): $p p o A$ fusion Fwd (5' ppoA HindIII/NcoI), ppoA fusion Rev (5' 23-bp ppoB, 23 bp рроA 3'), рроB Fusion Fwd (5' 23-bp pроA, 23-bp рроB $3^{\prime}$ ), and $p p o B$ Fusion Rev (3' ppoB BamHI/NcoI). The two fragments of $p p o A$ and $p p o B$ were fused by PCR using methods described by Yu and associates (2004) and subcloned into pCR2.1 TOPO vector (Invitrogen). The resulting fusion fragment contained engineered restriction sites: 5'ppoA HindIII/ NcoI and 3'ppoB AscI/BamHI. The nested sites NcoI and AscI were used to insert the fusion fragment in the forward orientation upstream of the gfp spacer fragment in pTMH44.2 to create pJBS5. Next, the outer restriction sites, HindIII and $B a m \mathrm{HI}$, were used to insert the fusion fragment in the reverse orientation to create pJBS5.1, which was sequenced through the region where each ppo fusion fragment meets pTMH44.2 (to confirm the orientation of each fusion fragment). For the ppoC::lox RNAi construct, gene fragments from $p p o C$ and lox, approximately 800 and $700 \mathrm{bp}$, respectively, in length with no predicted introns, were amplified using the primers ppoC fusion Fwd (5' ppoC) and ppoC::lox fusion Rev (25-bp 3' ppoC and 44-bp $5^{\prime} l o x$ ) for the $p p o C$ fragment. The pair of primers lox.fusion.for Fwd ( $5^{\prime}$ lox) and lox.fusion.rev ( $3^{\prime}$ lox) were used to amplify the lox fragment. The two fragments of lox and $p p o C$ were fused by PCR using methods described by $\mathrm{Yu}$ and associates (2004), then amplified by nested primers ppoC.nest.notI.ncoI.for and lox.nest.BamHI.AscI.rev. The resulting fusion fragment, ppoC::lox, contained engineered restriction sites at the $5^{\prime}$ ppoC not I/NcoI and 3' lox BamHI/AscI ends. The nested sites NcoI and AscI were used to insert the fusion fragment in the forward orientation upstream of the gfp spacer fragment in pTMH44.2 to create pSHB6.8. Next, the outer restriction sites BamHI and NotI were used to insert the fusion fragment in the reverse orientation to create $\mathrm{pSHB} 7.2$, which was sequenced through the region where each ppo fusion fragment meets pTMH44.2. Plasmids pJBS5.1 and pSHB7.2 were used for transformation of MJBS4.0.50 and TJBS5.1.60 to silence the expression of ppoA:ppoB and ppoC:lox, respectively.

\section{Fungal transformation procedure and mutants confirmation.}

Fungal transformation essentially followed that of Miller and associates (1985). The ppo deletion strains were obtained by a double-crossover event, exchanging the $p y r G$ selectable marker gene for the ppo coding region (Fig. 2A through $\mathrm{C}$ ). Aspergillus protoplasts were produced from freshly germinated conidia from 3-day-old cultures and transformed using a polyethylene glycol method (Miller et al. 1985). The DNA (5 to $10 \mu \mathrm{g}$ ) used for transformation was PCR amplified with nested primers from each disruption vector and gel purified using the QIAquick gel extraction kit (Qiagen, Hilden, Germany). Transformation with the $p p o A:: p p o B$ IRT vector pJBS5.1 was performed as a cotransformation with the niaD PCR product from A. flavus (primers listed in Table 3). Like- wise, transformation with the ppoC::lox IRT vector pSHB7.2 was performed as a cotransformation with the $\mathrm{pBC}$-Phleo plasmid carrying the phleomycin resistance cassette in which the ble gene is under the control of the A. nidulans gpdA promoter and the Saccharomyces cerevisiae CYC1 terminator (He et al. 2007). Transformants were screened for appropriate growth properties: uridine and uracil prototrophs for strains carrying a pyrG marker, nitrate prototrophs for strains with niaD, or resistance to phleomycin for strains carrying ble. Dot blots were made by spotting $3 \mu \mathrm{l}$ of genomic DNA from each transformant in a 1-cm grid on Hybond-XL membranes (Amersham Biosciences) and probed with an internal fragment of the appropriate ppo gene. Southern analysis performed in triplicate for each transformant was used to confirm a single gene replacement event in TJBS3.1.19, TJBS4.1.85, and TSHB2.2.15 (Fig. 2A through $\mathrm{C}$ ), probing each transformant with the $5^{\prime}$ flank and $3^{\prime}$ flank or the ORF of the appropriate ppo. Strains carrying the $p p o A: p p o B$ IRT vector pJBS5.1 were screened by dot blot in a fashion similar to ppo knockouts, and probed with the $g f p$ spacer fragment excised from pTMH44.2 using AscI and BamHI. Spotted isolates with strong $g f p$ hybridization were then selected for Southern analysis and probed with the same $g f p$ fragment. Additionally, to confirm the efficiency of ppoA and $p p o B$ transcript reduction, Northern analysis was performed using internal fragments of each gene from primers рроA Int For, $р р о A$ Int Rev, $р р о B$ Int For, and $p p o B$ Int Rev (Table 3). IRT4 strains carrying the ppoC:lox IRT vector in addition to the $p p o A:: p p o B$ IRT vector were confirmed by digestion with $N c o$ I and were screened for the presence of a 3.2$\mathrm{kb}$ band and contain the inverted repeat transgene. The ppoC:lox IRT strain carrying a single copy was called TSHB5.57. Northern analysis was performed using a DNA probe of internal fragments of each gene from primers ppoC Int For, ppoC Int Rev, lox Int For and lox Int Rev, ppoA Int For, ppoA Int Rev, and ActinF and ActinR for Actin-specific DNA probe (Table 3). Detection of signals was carried out with a Phosphorimager-SI (Molecular Dynamics).

\section{Statistical analysis.}

Data were analyzed using the JMP software package (version 3.2.6; SAS Institute, Inc, Cary, NC, U.S.A.). Mean comparisons of sclerotia and conidial formation affected by different conidia concentration or strains were calculated using least significant difference according to the Tukey-Kramer multiple comparison test at $P \leq 0.05$. Mean values with different letters are significant.

\section{ACKNOWLEDGMENTS}

We thank G. A. Payne for access to A. flavus genome data and J. Starr for peanut seed. This research was funded by BARD No. FI-384-2006 to S. H. Brown and National Science Foundation IOB-0544428, subagreement S060039 to N. P. Keller.

\section{LITERATURE CITED}

Bennett, J. W. 1979. Aflatoxin and anthraquinones from diploids of Aspergillus parasiticus. J. Gen. Microbiol. 113:127-136.

Bhatnagar, D., Carry, J. W., Ehrlich, K., Yu, J., and Cleveland, T. E. 2006. Understanding the genetics of regulation of aflatoxin production and Aspergillus flavus development. Mycopathologia 162:155-166.

Brodhagen, M., Tsitsigiannis, D. I., Hornung, E., Goebel, C., Feussner, I., and Keller, N. P. 2008. Reciprocal oxylipin-mediated cross-talk in the Aspergillus-seed pathosystem. Mol. Microbiol. 67:378-391.

Burow, G. B., Nesbitt, T. C., Dunlap, J., and Keller, N. P. 1997. Seed lipoxygenase products modulate Aspergillus mycotoxin biosynthesis. Mol. Plant-Microbe Interact. 10:380-387.

Burow, G. B., Gardner, H. W., and Keller, N. P. 2000. A peanut seed lipoxygenase responsive to Aspergillus colonization. Plant Mol. Biol. 42:689-701. 
Calvo, A. M., Hinze, L. L., Gardner, H. W., and Keller, N. P. 1999. Sporogenic effect of polyunsaturated fatty acids on development of Aspergillus spp. Appl. Environ. Microbiol. 65:3668-3673.

Calvo, A. M., Wilson, R. A., Bok, J. W., and Keller, N. P. 2002. Relationship between secondary metabolism and fungal development. Microbiol. Mol. Biol. Rev. 66:447-459.

Calvo, A. M., Bok, J. W., Brooks, W., and Keller, N. P. 2004. VeA is required for toxin and sclerotial production in Aspergillus parasiticus. Appl. Environ. Microbiol. 70:4733-4739.

Champe, S. P., and El-Zayat., A. A. 1989. Isolation of a sexual sporulation hormone from Aspergillus nidulans. J. Bacteriol. 171:3982-3988.

Champe, S. P., Rao, P., and Chang, A. 1987. An endogenous inducer of sexual development in Aspergillus nidulans. J. Gen. Microbiol. 133:1383-1387

Chen, H., and Fink, G. R. 2006. Feedback control of morphogenesis in fungi by aromatic alcohols. Genes Dev. 20:1150-1161.

Chen, H., Fujita, M., Feng, Q., Clardy, J., and Fink, G. R. 2004. Tyrosol is a quorum-sensing molecule in Candida albicans. Proc. Natl. Acad. Sci. U.S.A. 101:5048-5052.

Chenna, R., Sugawara, H., Koike, T., Lopez, R., Gibson, T. J., Higgins, D. G., and Thompson, J. D. 2003. Multiple sequence alignment with the Clustal series of programs. Nucleic Acids Res. 31:3497-3500.

Cotty, P. J. 1988. Aflatoxin and sclerotial production by Aspergillus flavus: Influence of PH. Phytopathology 78:1250-1253.

Cotty, P. J., and Mellon, J. E. 2006. Ecology of aflatoxin producing fungi and biocontrol of aflatoxin contamination. 22:110-117.

Cove, D. J. 1976. Chlorate toxicity in Aspergillus nidulans: The selection and characterization of chlorate resistant mutants. Heredity 36:191-203.

Dagenais, T., Chung, D., Giles, S., Hull, C., Andes, D., and Keller, N. P. 2008. Defects in conidiophore development and conidial/macrophage interactions in a dioxygenase mutant of Aspergillus fumigatus. Infect. Immun. 76:3214-20.

Diener, U. L., Cole, R. J., Sanders, T. H., Payne, G. A., Lee, L. S., and Klich, M. A. 1987. Epidemiology of aflatoxin formation by Aspergillus flavus. Annu. Rev. Phytopathol. 25:249-270.

Funk, C. D. 2001. Prostaglandins and leukotrienes: Advances in eicosanoid biology. Science 294:1871-1875.

Gao, X., Shim, W. B., Göbel, C., Kunze, S., Feussner, I., Meeley, R., Balint-Kurti, P., and Kolomiets, M. 2007. Disruption of a maize 9-lipoxygenase results in increased resistance to fungal pathogens and reduced levels of contamination with mycotoxin fumonisin. Mol. PlantMicrobe Interact. 20:922-933.

Gao, X., Brodhagen, M., Isakeit, T., Horowitz Brown, S., Gobel, C., Betran, J., Feussner, I., Keller, N. P., and Kolomiets, M. V. 2009. Inactivation of the lipoxygenase ZmLOX3 increases susceptibility of maize to Aspergillus spp. Mol. Plant-Microbe Interact. 22:222-231.

Garscha, U., Jernerèn, F., Chung, D. W., Keller, N. P., Hamberg, M., and Oliw, E. H. 2007. Identification of dioxygenases required for Aspergillus development: Studies of products, stereochemistry and the reaction mechanism. J. Biol. Chem. 282:34707-34718.

Gqaleni, N., Smith, J .E., Lacey, J., and Gettinby, G. 1997. Effect of temperature, water activity, and incubation time on production of aflatoxins and cyclopiazonic acid by an isolate of Aspergillus flavus in surface agar culture. Appl. Environ. Microbiol. 63:1048-1053.

Greene, A. V., Keller, N. P., Haas, H., and Bell-Pedersen, D. 2003. A circadian oscillator in Aspergillus spp. regulates daily development and gene expression. Eukaryot. Cell 2:231-237.

Guzmán-de-Peña, D., and Ruiz-Herrera, J. 1997. Relationship between aflatoxin biosynthesis and sporulation in Aspergillus parasiticus. Fungal Genet. Biol. 21:198-205.

He, Z. M., Price, M. S., Obrian, G. R., Georgianna, D. R., and Payne. G. A. 2007. Improved protocols for functional analysis in the pathogenic fungus Aspergillus flavus. BMC Microbiol. 7:104.

Hedayati, M. T., Pasqualotto, A. C., Warn, P. A., Bowyer, P., and Denning, D. W. 2007. Aspergillus flavus: Human pathogen, allergen and mycotoxin producer. Microbiology 153:1677-1692.

Horn, B. W. 2007. Biodiversity of Aspergillus section Flavi in the United States: A review. Food Addit. Contam. 24:1088-1101.

Horowitz Brown, S., Zarnowski, R., Sharpee, W. C., and Keller, N. P. 2008. Morphological transition governed by density dependent and lipoxygenase activity in Aspergillus flavus. Appl. Environ. Microbiol. 74:5674-5685.

Hyeon, B. 1976. Chemical studies on the factors controlling sporulation of fungi. Chem. Regul. Plants 11:69-76.
Keller, N. P., and Hohn, T. M. 1997. Metabolic pathway gene clusters in filamentous fungi. Fungal Genet. Biol. 21:17-29.

Klich, M. 2007. Aspergillus flavus: The major producer of aflatoxin. Mol. Plant Pathol. 8:713-722.

Liavonchanka, A., and Feussner, I. 2006. Lipoxygenases: Occurrence, functions and catalysis. J. Plant Physiol. 163:348-357.

McDonald, T., Devi, T., Shimizu, K., Sim, S.-C. and Keller, N. P. 2004 Signaling events connecting mycotoxin biosynthesis and sporulation in Aspergillus and Fusarium spp. Pages 139-147 in: New Horizon of Mycotoxicology for Assuring Food Safety. T. Yoshizawa, ed. Bikohsha. Co., Takamatsu, Japan.

McDonald, T., Brown, D., Keller, N. P., and Hammond, T. M. 2005. RNA Silencing of mycotoxin production in Aspergillus and Fusarium species. Mol. Plant-Microbe Interact. 18:539-545.

Miller, B. L., Miller, K. Y., and Timberlake, W. E. 1985. Direct and indirect gene replacements in Aspergillus nidulans. Mol. Cell. Biol. 5:1714-1721.

Nemchenko, A., Kunze, S., Feussner, I., and Kolomiets, M. 2006. Duplicate maize 13-lipoxygenase genes are differentially regulated by circadian rhythm, cold stress, wounding, pathogen infection, and hormonal treatment. J. Exp. Bot. 57:3767-3779.

Nukima, M., Sassa, T., Ikeda, M., and Takahashi. K. 1981. Linoleic acid enhances perithecial production in Neurospora crassa. Agric. Biol. Chem. 45:2371-2373.

Robens, J., and Cardwell, K. 2003. The cost of mycotoxin management to the USA: Management of aflatoxins in the United States. J. Toxicol. Toxin Rev. 22:143-156.

Sambrook, J., Fritsch, E. F., and Maniatis, T. 1989. Molecular Cloning: A Laboratory Manual. Cold Spring Harbor Laboratory Press, Cold Spring Harbor, NY, U.S.A..

Scheidegger, K. A., and Payne, G. A. 2003. Unlocking the secrets behind secondary metabolism: A review of Aspergillus flavus from pathogenicity to functional genomics. J. Toxicol. Toxin Rev. 22:423-459.

Shea, J. M., Henry, J. L., and Del Poeta, M. 2006. Lipid metabolism in Cryptococcus neoformans. Antimicrob. Agents Chemother. 6:469-479.

Shimizu, K., and Keller, N. P. 2001. Genetic involvement of a cAMP-dependent protein kinase in a $\mathrm{G}$ protein signaling pathway regulating morphological and chemical transitions in Aspergillus nidulans. Genetics 157:591-600.

Sprague, G. F., and Winans, S. C. 2006. Eukaryotes learn how to count: Quorum sensing by yeast. Genes Dev. 20:1045-1049.

Tsitsigiannis, D. I., and Keller, N. P. 2006. Oxylipins act as determinants of natural product biosynthesis and seed colonization in Aspergillus nidulans. Mol. Microbiol. 59:882-892.

Tsitsigiannis, D. I., and Keller, N. P. 2007. Oxylipins as developmental and host-fungal communication signals. Trends Microbiol. 15:109-118.

Tsitsigiannis, D. I., Kowieski, T. M., Zarnowski, R., and Keller, N. P. 2004. Endogenous lipogenic regulators of spore balance in Aspergillus nidulans. Eukaryot. Cell 3:1398-1411.

Tsitsigiannis, D. I., Bok, J. W., Andes, D., Nielsen, K. F., Frisvad, J. C., and Keller, N. P. 2005a. Aspergillus cyclooxygenase-like enzymes are associated with prostaglandin production and virulence. Infect. Immunol. 8:4548-4559.

Tsitsigiannis, D. I., Kowieski, T. M., Zarnowski, R., and Keller, N. P. 2005b. Three putative oxylipin biosynthetic genes integrate sexual and asexual development in Aspergillus nidulans. Microbiology 151:1809-1821.

Yu, J. H., Hamari, Z., Han, K. H., Seo, J. A. Dominguez, Y. R., and Scazzocchio, C. 2004. Double-joint PCR: A PCR-based molecular tool for gene manipulations in filamentous fungi. Fungal Genet. Biol. 41:973-981.

Wicklow, D. T., Horn, B. W., and Cole, R. J. 1982. Sclerotium production by Aspergillus flavus on corn kernels. Mycologia 74:398-403.

\section{AUTHOR-RECOMMENDED INTERNET RESOURCES}

National Center for Biotechnology Information Conserved Domain search page: www.ncbi.nlm.nih.gov/Structure/cdd/wrpsb.cgi

San Diego Supercomputer Center Biology Workbench tool: workbench.sdsc.edu

Image J software: rsb.info.nih.gov/ij/index.htm

EMBL-EBI ClustalW multiple sequence alignment program www.ebi.ac.uk/clustalw

The Center for Integrated Fungal Research Aspergillus flavus website: www.aspergillusflavus.org 